\section{ANNUAL Further}

Click here to view this article's online features:

- Download figures as PPT slides

- Navigate linked references

- Download citations

- Explore related articles

- Search keywords

\title{
Experimental Milestones in the Discovery of Molecular Chaperones as Polypeptide Unfolding Enzymes
}

\author{
Andrija Finka,,$^{1, *}$ Rayees U.H. Mattoo, ${ }^{2}$ \\ and Pierre Goloubinoff ${ }^{3, * *}$
}

\author{
${ }^{1}$ Laboratory of Biophysical Statistics, School of Basic Sciences, Ecole Polytechnique Fédérale de \\ Lausanne, CH-1015 Lausanne, Switzerland \\ ${ }^{2}$ Department of Structural Biology, Stanford University, Stanford, California 94305; \\ email: rmattoo@stanford.edu
}

${ }^{3}$ Department of Plant Molecular Biology, Faculty of Biology and Medicine, University of Lausanne, CH-1015 Lausanne, Switzerland; email: pierre.goloubinoff@unil.ch

Annu. Rev. Biochem. 2016. 85:715-42

First published online as a Review in Advance on March 31, 2016

The Annual Review of Biochemistry is online at biochem.annualreviews.org

This article's doi:

10.1146/annurev-biochem-060815-014124

Copyright (c) 2016 by Annual Reviews. All rights reserved

*Present address: Department of Ecology, Agronomy and Aquaculture, University of Zadar, HR-23000 Zadar, Croatia; email: afinka@unizd.hr

${ }^{* *}$ Corresponding author.

\section{Keywords}

heat-shock proteins, Hsp60, Hsp70, Hsp110, Hsp104, small heat-shock proteins, sHsps, protein homeostasis, unfoldases

\begin{abstract}
Molecular chaperones control the cellular folding, assembly, unfolding, disassembly, translocation, activation, inactivation, disaggregation, and degradation of proteins. In 1989, groundbreaking experiments demonstrated that a purified chaperone can bind and prevent the aggregation of artificially unfolded polypeptides and use ATP to dissociate and convert them into native proteins. A decade later, other chaperones were shown to use ATP hydrolysis to unfold and solubilize stable protein aggregates, leading to their native refolding. Presently, the main conserved chaperone families Hsp70, Hsp104, Hsp90, Hsp60, and small heat-shock proteins (sHsps) apparently act as unfolding nanomachines capable of converting functional alternatively folded or toxic misfolded polypeptides into harmless protease-degradable or biologically active native proteins. Being unfoldases, the chaperones can proofread three-dimensional protein structures and thus control protein quality in the cell. Understanding the mechanisms of the cellular unfoldases is central to the design of new therapies against aging, degenerative protein conformational diseases, and specific cancers.
\end{abstract}




\section{Contents}

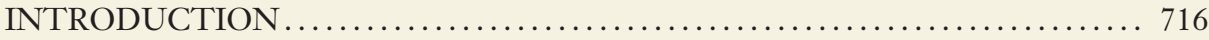

THE PREEXPERIMENTAL ERA: THE BIRTH OF THE MOLECULAR

CHAPERONE CONCEPT ...................................... 717

THE FIRST EXPERIMENTAL PROOF OF THE

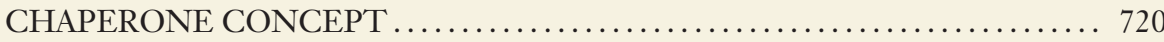

A Molecular Chaperone Can Favor Native Folding of a Foreign

Protein in the Cell.......................................... 721

A Purified Molecular Chaperone Can Prevent Protein

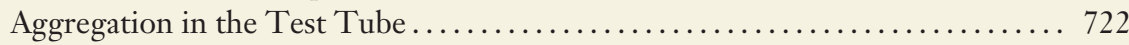

A Purified Molecular Chaperone Can Use ATP to Dissociate Bound Polypeptides

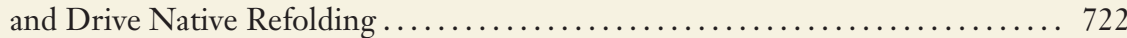

Chaperones Can Unfold Misfolded Polypeptides ...................... 724

Chaperones Can Disaggregate Large Insoluble Aggregates ................. 727

Chaperones Can Catalytically Unfold Stable Misfolded Polypeptides........... 732

CHAPERONE ACTIVITY DOES NOT DEPEND ON IN-CAGE

CONFINEMENT OF FOLDING INTERMEDIATES ................ 733

MOLECULAR CHAPERONES SHOULD REVERT TO THEIR

ORIGINAL NAME: UNFOLDING ENZYMES .................... 734

THE UNFOLDASES CAN PROOFREAD THREE-DIMENSIONAL PROTEIN

STRUCTURES AND THEREBY CONTROL PROTEOSTASIS .......... 735

THE IMPORTANCE OF UNDERSTANDING POLYPEPTIDE UNFOLDASE

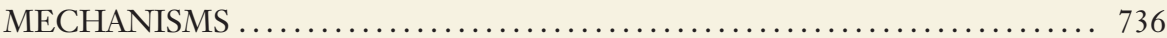

Unfolding enzymes or unfoldases: enzymes that can convert by unfolding high-affinity misfolded or alternatively folded polypeptide substrates into low-affinity unfolded and natively refolded products

Alternatively folded protein: a native protein that can be converted by an unfolding enzyme (chaperone) into a differently active native protein

\section{INTRODUCTION}

In the crowded environment of the cell, molecular chaperones control all aspects of protein homeostasis. Recent findings show that the main conserved chaperone families, Hsp70/Hsp110 (DnaK), Hsp104 (ClpB), Hsp90s (HtpG), Hsp60/CCT (GroEL), and the small heat-shock proteins (sHsps) (IbpA/B) (traditional prokaryotic names in brackets), can specifically target and unfold alternatively folded, or stably misfolded and aggregated conformers, which may be cytotoxic, and convert them into harmless functional native proteins or degraded peptides. All chaperones seem to share a common mode of action involving cycles of substrate binding, unfolding, dissociation, and spontaneous refolding, or protease-mediated degradation (see sidebar, About Heat-Shock Proteins and Molecular Chaperones). In 1986, Rothman \& Kornberg (1) initially suggested the biomechanistically informative term unfolding enzymes. Yet, a year later, the socially inspired term molecular chaperone, which is scientifically less accurate, was adopted by the scientific community (2).

Indicative of their central role, the chaperones/unfoldases can be up to $10 \%$ of the total protein mass of human cells $(3,4)$. By virtue of their ability to specifically unfold a minority of misfolded and alternatively folded proteins in the cell, the chaperones/unfoldases can proofread the threedimensional structures of the remaining $90 \%$ of the cellular proteins and control their conformational quality during their eventful and stressful life. The unfolding enzymes may thus effectively reduce the concentrations of stress- or mutation-induced pathogenic protein conformers, which cause the late-age onset of many degenerative diseases in animals. 


\section{ABOUT HEAT-SHOCK PROTEINS AND MOLECULAR CHAPERONES}

In 1974, specific proteins of various molecular weights were found to massively accumulate in cells under heat shock. They were named heat-shock proteins (Hsps) and classified according to their size on sodium dodecyl sulfate gels (28). Because bacterial GroEL and yeast mitochondrial Hsp60 happened to be among them (these were also highly homologous to the first-characterized molecular chaperone, chloroplast RuBisCO-binding protein), all similar proteins were generally called Hsp60s. Similarly, because specific members in each class of molecular chaperones were also Hsps, all members became generally called Hsp70s, Hsp110s, Hsp100s, Hsp90s, and small Hsps (sHsps). A simple bioinformatic homology search of the human genome shows 39 core chaperone genes [13 for the Hsp70s, 5 Hsp90s, 10 chaperonins, and 11 sHsps (137)]; we found that only 15 of them (7 Hsp70s, 3 Hsp90s, and 5 chaperonins) are significantly overexpressed in heat-shocked human cells at $41^{\circ} \mathrm{C}$. Thus, the remaining 24 chaperones are faultily named Hsps $(4,120)$ and would have benefited from being called Unfoldase 70 , Unfoldase 90, Unfoldase 60 and small unfoldases, respectively.

\section{THE PREEXPERIMENTAL ERA: THE BIRTH OF THE MOLECULAR CHAPERONE CONCEPT}

The central dogma of molecular biology describes the sequential flow of biological information, principally from DNA-based genes that are transcribed into messenger RNAs (mRNAs). The mRNAs are in turn translated into polypeptide chains that need to translocate, properly fold, and assemble into compact dynamic protein complexes in order to carry out specific biological functions (5). Ultimately, proteins need to be timely degraded and recycled. Over the years, major milestones on this flowchart have been acknowledged: Crick, Watson, and Wilkins were awarded the Nobel prize for the structure of the DNA double helix; A. Kornberg for the structure of DNA polymerase and the mechanism of DNA replication; Modrich, Lindahl, and Sancar for the mechanisms of DNA repair (6); R. Kornberg for the structure of RNA polymerase and the mechanism of transcription; and Ramakrishnan, Steitz, and Yonath for the structure of the ribosome and the mechanism of translation. Anfinsen was acknowledged for finding that artificially unfolded polypeptides can spontaneously refold to their native state, and Prusiner for unveiling the toxic and infectious nature of misfolded proteins. Rose, Ciechanover, and Hershko were rewarded for discovering that protein degradation is an energy-consuming process that is strictly controlled by ubiquitin tagging (7). It is noteworthy that the molecular chaperones that control a plethora of processes in cellular protein homeostasis still lack full recognition (Figure 1).

The main highly conserved chaperones families, Hsp60s (GroEL), Hsp70/Hsp110s (DnaK), Hsp104s (ClpB), Hsp90s (HtpG), and the sHsps (IbpA/B) (bacterial chaperones are in brackets), can constitute up to $10 \%$ of the total protein mass of cultured human cells (3). They tightly control the de novo folding, unfolding, assembly/disassembly, translocation, activation/inactivation, disaggregation, and degradation of proteins in the different cellular compartments (8, 9). Anfinsen et al. (10) demonstrated that the primary amino acid sequence of a polypeptide may, in principle, contain all the necessary information for it to reach its native three-dimensional conformation without necessitating assistance, or steric information, from other macromolecules. Yet, Anfinsen and colleagues (11) also observed that in vitro protein-refolding assays were often inefficient and suggested that other proteins might influence the protein folding process in the cell. The effective protein concentration in typical cultured human cells may reach about $150 \mathrm{mg} / \mathrm{mL}$ (3), implying a dense crowding of about a million polypeptides per cubic micrometer (12), commonly thought to favor the misfolding and aggregation, especially of nascent multidomain polypeptides $(13,14)$.

Unfolded polypeptide: usually an unstable uncompacted polypeptide chain lacking secondary structures, generally has a low affinity for unfolding enzymes (chaperones) 


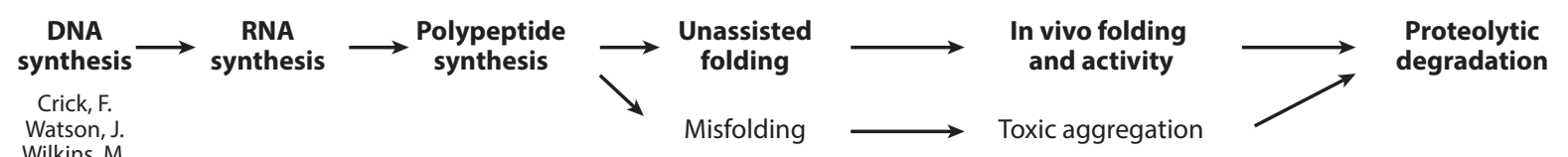

Wilkins, M.

Kornberg, A.

Modrich, $\mathrm{P}$.

Lindahl, $\mathrm{T}$.

Sancar, A.

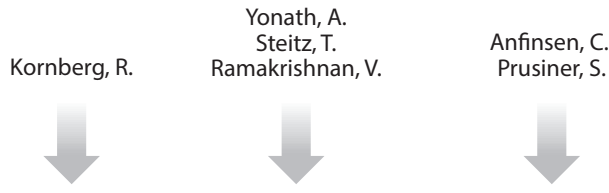

Anfinsen, C.

Ciechanover, A. Hershko, A. Rose, I.
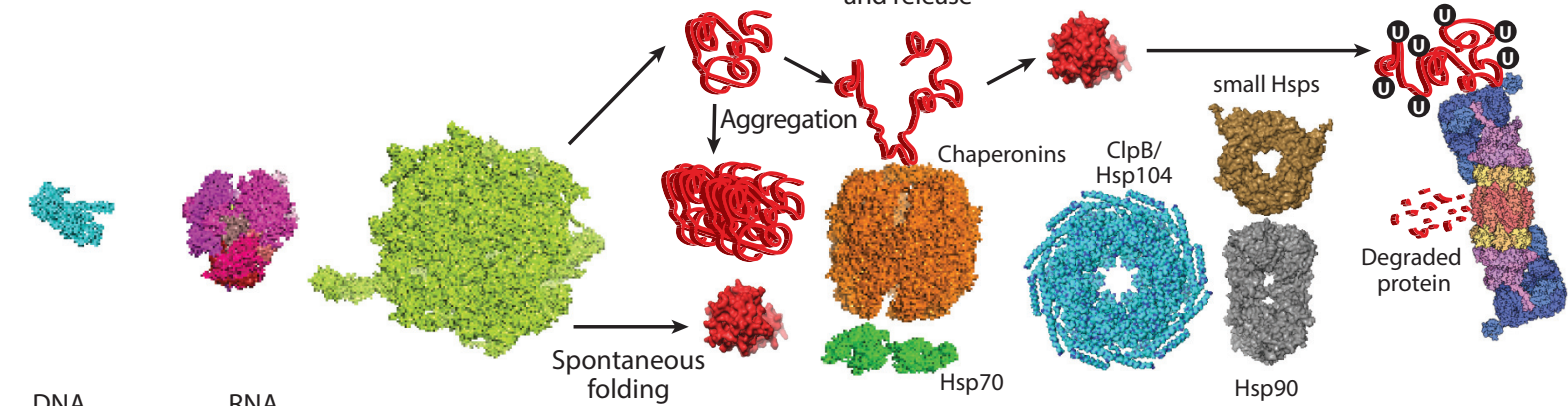

Misfolded
protein
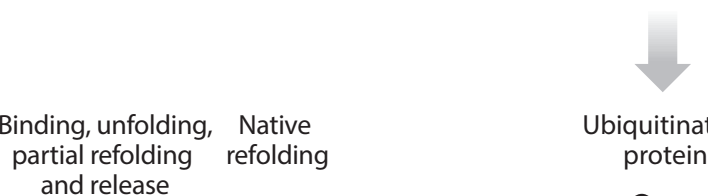

Ubiquitinated protein

DNA

RNA polymerase polymerase

Ribosome

Foldases and molecular chaperones

Proteases

\section{Figure 1}

Important steps in the pathway from DNA to protein degradation have been internationally recognized. (Top) Nobel prizes given for major findings (gray arrows) on this flowchart for DNA synthesis, RNA synthesis, protein synthesis, protein folding, prion propagation, and protein degradation. (Middle) The molecular machines involved in each step. (Bottom) The cellular processes that are carried out by the above molecular machines. Still lacking full recognition are the molecular chaperones that control de novo folding and assembly of native proteins, the activation/inactivation of signaling proteins, the translocation of polypeptides to organelles, and protein secretion. Importantly, by virtue of their ability to specifically unfold misfolded conformers, chaperones can proofread the three-dimensional structure of proteins and thus mediate various cellular functions and control protein quality under stress and aging. Abbreviation: Hsp, heat-shock protein.

The first genetic indication that particular proteins may control the assembly of various large protein complexes in cells came in 1973 with the characterization of Escherichia coli mutants that were defective in bacteriophage maturation (15). Point mutations in the bacterial genes GroES, GroEL, DnaK, Dnaf, and GrpE impaired the maturation of bacteriophage capsids and tails $(16,17)$, suggesting that the bacterial gene products, GroEL in particular, could carry an assembly aiding function (18). Negative-stain electron microscopy showed that the GroEL protein was made of fourteen $60-\mathrm{kDa}$ identical subunits assembled in two stacked heptameric rings (18), a uniquely recognizable size and shape that later led to the identification of the GroEL orthologs: Cpn60 in chloroplasts and Hsp60 in mitochondria.

A second indication that a nascent protein, although generally able to fold spontaneously into a native monomer, might still need an assembly-aiding protein complex to fold and assemble into a functional oligomer in the crowded environment of cells came in 1980 from isolated chloroplasts. Using pulse-chase experiments and autoradiograms of nondenaturing and denaturing gels, Barraclough \& Ellis (19) showed that nascent radiolabeled 55-kDa ribulose-1,5-biphosphate carboxylase oxygenase (RuBisCO) large subunits (LSUs) synthesized on chloroplast ribosomes did first transiently associate in the chloroplast stroma to an abundant $\sim 700-\mathrm{kDa}$ complex made of $60-\mathrm{kDa}$ subunits, before it later integrated mature 520-kDa RuBisCO holoenzymes. 
The term molecular chaperone was originally coined by Laskey et al. (20) in 1978 to describe the ability of nucleoplasmin to prevent inappropriate electrostatic interactions between histones and oppositely charged proteins. In 1987, Ellis and colleagues $(2,21)$ adapted this term to describe the alleged biochemical function of a new class of proteins that seemingly assisted the proper assembly of large protein oligomers, such as RuBisCO and phages, by a yet unknown mechanism involving noncovalent binding of the nascent polypeptide chains and their subsequent ATP-dependent dissociation. Although unscientifically borrowed from human sociology, the term chaperone initially fit what appeared to be at the time the main molecular function of these proteins: the mere binding of misfolding polypeptides impairing their aggregation, a passive biological function performed by very abundant proteins, which appeared quite wasteful as they were noncatalytic.

Because the defective phage maturation phenotype in DnaK E. coli mutants was suspected to result from an impaired replication of phage $\operatorname{DNA}(16,22)$, a first biochemical assay was developed by Georgopoulos and colleagues (22), who showed that purified DnaK and DnaJ in the presence of ATP could collaborate with other proteins in the facilitation of bacteriophage $\lambda$ DNA replication in vitro (24-26). It is noteworthy that this seminal in vitro assay with purified DnaK and DnaJ proteins did not reveal any information about a possible mechanism of action, especially in relation to what was later called chaperone activity. Five more years were necessary for the first in vitro chaperone activity assay to be designed with the specific aim of addressing the ability of a given protein, GroEL, to prevent the aggregation of artificially denatured proteins and subsequently mediate their proper native refolding and assembly in strict ATP- and GroES-dependent manners (27).

In 1974, Tissières et al. (28) described the first sodium dodecyl sulfate gel and autoradiogram of heat-shock proteins (Hsps) labeled with ${ }^{35} S$-methionine, which were seen to accumulate in heat-shocked salivary glands of Drosophila in relation to chromosome puffs. This initiated the general use of the term Hsp, associated with apparent molecular weights, without speculating on possible molecular functions (28). A decade later, Varshavsky and colleagues (29) suggested that Hsps in general might bind to denatured or abnormal proteins after a heat shock to prevent their aggregation and thus avert cellular damage. This was further developed in 1986 by Pelham (30), who predicted possible roles of Hsp70 and Hsp90 in a range of protein assembly and disassembly processes in the cell. On the basis of in vivo observations that Hsp70 molecules could become tightly associated to nucleoli under heat shock and that in vitro addition of ATP could revert this association, Lewis \& Pelham (31) hypothesized that in unstressed cells Hsp70s and Hsp90s might bind to rare proteins that transiently expose hydrophobic surfaces during their natural folding and assembly processes. In stressed cells, it was suggested that Hsp70s and Hsp90s were required in increased amounts to dissociate aggregates and prevent further aggregation (30).

At the same time, Rothman \& Kornberg (1) published a plainly visionary News and Views paper in which, commenting on the work of Eilers \& Schatz (32) on Hsp70-mediated protein translocation across the mitochondrial import pore and the work of Waxman \& Goldberg (33) on protein translocation into the protease chamber of the Lon $(\mathrm{La})$ protease, they suggested the existence of unfolding enzymes. These unfolding enzymes were to control cellular processes, such as translocation and unfolding of polypeptides across membranes, the forceful unfolding of misfolded proteins, and their refolding or their controlled degradation by proteases (1). Without mentioning the term chaperone that was not yet reinstated by Ellis, they wrote:

The power of unfolding enzymes could also be harnessed for constructive purposes. Such enzymes could even catalyze the fundamental process of protein folding. Although this process is generally viewed as spontaneous, because isolated polypeptides can refold efficiently, artificial maneuvers such as the gradual removal of a denaturing agent are often required. Wrongly folded structures otherwise form and persist or are degraded. Misfolded proteins in a cell might be recognized by virtue of the
Heat-shock proteins (Hsps): proteins that accumulate in cells during a heat shock; Hsp is often misused to generally describe a molecular chaperone 
chain they expose and then be actively unfolded to allow the folding process to repeat itself rapidly until a compact structure of minimum energy is achieved. Such catalysis of folding by unfolding enzymes would set a minimum stability for a correctly folded structure. (1, p. 210)

Nearly three decades later, these precise words describe most accurately the mechanistic denominator, which we find to be shared by the main families of conserved molecular chaperones.

At about the same time, Hemmingsen \& Ellis (21) purified the $\sim 700-\mathrm{kDa}$ RuBisCO-binding protein from the pea chloroplasts and found it to be composed of equal amounts of $60-\mathrm{kDa} \alpha-$ and $\beta$-subunits. Addition of ATP weakened the stability of the chloroplast oligomer and caused the apparent dissociation of bound radioactive ghosts of $55-\mathrm{kDa}$ RuBisCO, suggesting that the ATP-mediated deoligomerization of the chaperone complex caused the dissociation of the noncovalently associated RuBisCO polypeptides $(34,35)$. Later work with GroEL, the orthologous protein from bacteria, showed that, although ATP is involved in the release of the bound polypeptides, the release mechanism does not involve the disruption of the chaperone holo-oligomer (27). In 1988, Picard et al. (36) showed evidence that association of another Hsp, Hsp90, may inactivate the glucocorticoid receptor by keeping it unfolded, and in their seminal review on Hsps, Lindquist \& Craig (37) reiterated Rothman \& Kornberg's original idea (1) that, although lacking experimental evidence, Hsp70 proteins could be acting as cellular unfolding enzymes, altering the conformation of proteins in an ATP-dependent manner that could drive passage of polypeptides through membranes. Also in 1988, Girshovich and colleagues (38) used photocross-linking to show that newly synthesized polypeptides in E. coli cells could transiently bind to GroEL complexes and that ATP could dissociate these complexes in vitro. They hypothesized that GroEL might have a particular affinity for unfolded nascent proteins and could use the energy of ATP hydrolysis to impede aggregation. Work a decade later showed that another passive chaperone, trigger factor, at the bacterial ribosome exit could also secure the proper folding of nascent chains in collaboration with the ATP-fueled action of Hsp70 (39).

In earlier work using negative-stain electron microscopy, Pushkin et al. (40) observed the presence of high-molecular-weight protein complexes in soluble extracts of pea chloroplasts that were identical in size and displayed the same double-stacked sevenfold symmetry as the GroEL $\mathrm{L}_{14}$ complexes from E. coli (40). Having completed the sequencing of the $\alpha$ - and $\beta$-subunits of the RuBisCO-binding proteins, this triggered in 1988 Hemmingsen and Ellis to contact C. Georgopoulos, who had recent knowledge of the sequence of E. coli GroEL. Primary amino acid sequence alignments showed an astoundingly high level of sequence identity between the bacterial GroEL and the two plant $\alpha$ - and $\beta$-RuBisCO-binding protein subunits from pea (41). This was compelling correlative evidence that chloroplast RuBisCO-binding proteins and bacterial GroEL should perform similar biological functions, related to aiding the assembly of large protein complexes, by a mechanism involving transient noncovalent binding of polypeptides, followed by their ATP-dependent dissociation (2). However, this conclusion, which was based solely on highsequence homology, remained speculative and called for interventional experimental validations both in living cells and in the test tube with purified chaperone proteins and model polypeptide substrates.

\section{THE FIRST EXPERIMENTAL PROOF OF THE CHAPERONE CONCEPT}

Thus far, the birth of the chaperone concept was based on observations in bacteria and chloroplasts of possible correlations between the presence of one protein, GroEL or the RuBisCObinding protein, and the successful assembly of another protein complex, RuBisCO or phage. 
Interventional experiments were thus needed to convert correlative observations into experimental proofs that chaperone-assisted assembly of protein complexes was a general principle, possibly deserving a post of its own on the general flowchart of molecular biology (Figure 1). The possible experimental approaches were simple: Chaperone levels could be either artificially increased or decreased in the cell or in the test tube, and the effects could be tested on the quality of folding/ misfolding and assembly/aggregation of reporter substrate proteins. For example, at this stage the RuBisCO-binding protein and GroEL were thought to act predominantly on the assembly of large protein oligomers (41). It took a decade of biochemical studies with purified chaperones to gather convincing evidence that GroEL, as well as other classic chaperones, primarily acted directly on the folding/unfolding of individual polypeptide subunits.

\section{A Molecular Chaperone Can Favor Native Folding of a Foreign Protein in the Cell}

The first experimental evidence that a molecular chaperone can control the fate of protein folding and oligomer assembly in the highly crowded environment of a cell was demonstrated by Goloubinoff and colleagues (42) in whole E. coli bacteria harboring a plasmid that expressed the RuBisCO large and small subunits (LSUs and SSUs) from cyanobacteria under a constitutive promoter. The cyanobacterial LSU and SSU were well expressed; however, in the presence of only the low basal levels of GroES and GroEL expressed from the bacterial chromosome, they ended up being mostly inactive and insoluble in inclusion bodies. In contrast, when a compatible multicopy plasmid was present that ectopically expressed medium levels of additional GroES and GroEL proteins, the cyanobacterial LSU and SSU polypeptides were found to be mostly soluble and assembled into active hexadecameric $\left(\mathrm{L}_{8} \mathrm{~S}_{8}\right)$ holoenzymes. Stressing the importance of the GroES cochaperone, ectopic overexpression of only GroEL remained ineffective at promoting the formation of soluble cyanobacterial RuBisCO holoenzymes. The E. coli strains harboring missense mutations in GroES and GroEL, which were defective in phage assembly, also failed to optimally assemble dimeric recombinant RuBisCO from the purple bacteria Rhodospirillum rubrum (42). Interestingly, these in vivo proof-of-the-chaperone concept experiments also demonstrated the ubiquitous nature of the molecular chaperones. GroEL and GroES from E. coli seemed to apply a specific mechanism consisting of preventing the aggregation and promoting native assembly of phage head and tail proteins, which were optimal substrates for E. coli chaperones; however, GroEL and GroES also acted on nonself recombinant proteins from an evolutionarily more distant organism, albeit this was done with a lesser efficiency. Whereas the endogenous basal levels from the chromosomal GroES and GroEL genes sufficed to mediate optimal folding of dimeric RuBisCO from the gramnegative purple bacteria $R$. rubrum (a proteobacteria, which is a close relative of $E$. coli), massive overexpression of plasmid-encoded E. coli GroES and GroEL chaperones was necessary to optimally fold and assemble hexadecameric $\mathrm{RuBisCO}$ from cyanobacteria, which is evolutionarily much more distant from E. coli.

In support of this first proof-of-the-chaperone concept in whole living cells, Horwich and collaborators $(43,44)$ then showed that when various polypeptides generated on cell-free ribosomes were artificially imported into isolated yeast mitochondria harboring a thermosensitive point mutation in Hsp60, the GroEL ortholog, they showed different degrees of sensitivity to partial proteolysis, as compared with mitochondria expressing wild-type Hsp60. At this stage, the main molecular action of bacterial GroEL, yeast Hsp60, and chloroplast RuBisCO-binding protein was thought to be at the level of multisubunit complex assembly. Confirming the initial observations with isolated chloroplasts, this semi-in vivo system with isolated yeast mitochondria identified Hsp60 as a large complex that can also transiently interact with de novo imported polypeptides 
Aggregated

polypeptide:

an inactive, potentially toxic, oligomeric polypeptide enriched in nonnative intra- and intermolecular $\beta$-sheets, prone to form larger insoluble oligomers and determine their fate as misfolded or presumably native, as inferred from the greater protease sensitivity of the imported polypeptides in the Hsp60 mutants, as compared with the wild-type mitochondrial extracts (43-46).

\section{A Purified Molecular Chaperone Can Prevent Protein Aggregation in the Test Tube}

Even though the in vivo experiments with GroEL, GroES, and RuBisCO in whole cells were groundbreaking, the ultimate proof-of-the-chaperone concept called for the design of a purely in vitro activity assay, comprising only chaperones, ATP, and model polypeptides as substrates free of possible interferences from thousands of unrelated cellular proteins, nucleotides, membranes, and metabolites. Only such an in vitro activity assay could address in a rigorous dose-dependent manner the specific chaperone properties, such as their apparent ability to prevent protein aggregation and use ATP hydrolysis to convert inactive and aggregated polypeptides into active, natively folded, and assembled proteins. This founding experimental milestone in the field of molecular chaperones was crossed in 1989 by Goloubinoff and colleagues (27), who designed an assay with only pure GroEL and GroES proteins and RuBisCO as a model polypeptide substrate. More informative than mere differences in protease sensitivity, they used enzymatic activity as a proxy to assess the native folding of a polypeptide substrate before, during, and following chaperone action (27). For this purpose, purified native $R$. rubrum $\mathrm{RuBisCO}$ dimers were either completely unfolded by urea (or by guanidinium chloride) or misfolded by acid treatment. Circular dichroism showed that whereas urea-denatured RuBisCO was, as expected, completely devoid of secondary structures, the acid-denatured $\mathrm{RuBisCO}$ still contained many secondary structures, mostly misfolded $\beta$-sheets (27). Upon dilution of the urea, or dilution and neutralization of the acid, active $\mathrm{RuBisCO}$ did not spontaneously refold by itself but rather precipitated in the form of inactive insoluble aggregates. When, however, GroEL was present during dilution and neutralization of the denaturing agents, the $\mathrm{RuBisCO}$ polypeptides remained soluble but stayed inactive. Contrary to large aggregates that did not enter the native gels, the soluble inactive RuBisCO species were found tightly associated to the large soluble $840-\mathrm{kDa}_{\text {GroEL }} 14$ oligomers, with which they comigrated in the native gels (27). This was the first direct experimental evidence that a molecular chaperone can spontaneously associate tightly, albeit noncovalently, with misfolding or misfolded inactive polypeptide substrates, thereby preventing their aggregation, whereas native $\mathrm{RuBisCO}$ did not bind to the chaperone complex and rapidly migrated in the native gel as a free $110-\mathrm{kDa}$ dimer (27).

\section{A Purified Molecular Chaperone Can Use ATP to Dissociate Bound Polypeptides and Drive Native Refolding}

ATP added to chloroplast or bacterial extracts was already known to cause an apparent dissociation of chaperone-bound inactive $\mathrm{RuBisCO}$ or nascent polypeptides, but there was no information on the fate of the released species $(38,47)$. The first in vitro assay with pure components revealed the fate of the chaperone-released species: They were driven to properly refold and assemble into native proteins! Hence, addition of GroES and ATP to the preformed soluble GroEL $\mathrm{L}_{14}-$ $\mathrm{RuBisCO}$ complexes caused the dissociation of the bound inactive RuBisCO polypeptides and their refolding into enzymatically active, soluble RuBisCO dimers (27). This experiment thus showed that chaperones can use two sequential complementary mechanisms: In the first, which does not need ATP, the chaperones may spontaneously bind and prevent the aggregation of denatured polypeptides, and in the second, which requires ATP, the bound denatured polypeptides 
are forcefully dissociated in a controlled manner such that they may properly refold and assemble into native oligomers.

Shortly thereafter, an important control experiment was performed by Viitanen et al. (48). At low temperature and at dilute concentrations, the same urea-unfolded RuBisCO species were found to refold rather efficiently into the native state in the total absence of chaperones. Thus, under the ideal conditions of low crowding and low temperatures, the general principle set by Anfinsen that polypeptides should be able to fold unassisted by other proteins was also applicable to R. rubrum RuBisCO: Although the folding process can be viewed as spontaneous, accidents may happen at high temperatures. As predicted by Anfinsen, wrongly folded structures may form and call in the crowded environment of cells for the special assistance of molecular chaperones. This raised the question of the precise mechanism by which the chaperones could assist the spontaneous folding pathway of proteins. Was this a passive role, similar to that of a security fence bordering a vertiginous trail? It could also be a more active enzymatic unfolding role in which, similar to a crane, chaperones might lift misfolded polypeptides that went astray back onto the native folding path, acting as ATP-fueled unfolding enzymes. The very first in vitro refolding experiments provided significant clues on the molecular mechanisms used by GroEL and GroES. The dependence of the reaction on ATP hydrolysis was a clear indication that it involved more than passive binding to prevent substrate aggregation. It argued against using the social term chaperone, which is a rather passive social function of preventing aggregation by being there doing nothing, to describe such an ATP-dependent molecular function. The GroEL system was nearly as effective at refolding the acid-denatured $\mathrm{RuBisCO}$, which initially contained stable misfolded secondary structures, as it was to refold urea-denatured $\mathrm{RuBisCO}$, which initially lacked all secondary structures (27). This raised the possibility that in the case of the urea-unfolded RuBisCO, GroEL also acted on misfolded intermediate species that readily formed upon urea dilution rather than on the unfolded polypeptides per se, as generally believed, until today. This suggested that upon binding and hydrolyzing ATP, GroEL's role could be to forcefully destabilize the already formed misfolded secondary structures in the bound acid-denatured RuBisCO misfolded species, acting as an ATPfueled unfolding/stretching nanomachine (49-51). This did not exclude the possibility that GroEL could also act as a passive sequestering cage, especially during heat shock (52) in which unfolded polypeptides would be able to safely reach their native state, rather than obligatorily aggregate as when totally free in solution $(49,53)$.

The observations that purified GroEL can bind and prevent aggregation and that ATP with GroES can cause the dissociation of the bound polypeptides, leading to native refolding (27), were reiterated and generalized with other polypeptide substrates, such as guanidinium-HCl-denatured rhodanese, dihydrofolate reductase, ornithine transcarbamylase (54), citrate synthase (55), malate dehydrogenase (56), or acid ( $\mathrm{pH}$ 2) denatured cis-aconitase (57).

About a year after GroEL, GroES, and ATP were shown to drive the native refolding of denatured RuBisCO, a large molar excess of bacterial Hsp70 (DnaK) was shown to drive, in a strict ATP-dependent manner, the native refolding of heat-predenatured RNA polymerase (58). Although very inefficient at first, this reaction was later improved by the addition of the cochaperones DnaJ and GrpE $(59,60)$. It demonstrated that other proteins could act similarly to GroEL in binding misfolded polypeptides and preventing their aggregation and that ATP could promote substrate dissociation and native refolding. It is worth noting that DnaK is not known to form a sequestering cage, implying that in-cage sequestration of denatured polypeptides to prevent aggregation is not a general mechanism applicable to all chaperones. This also raised the possibility that not all denatured polypeptides are obligatorily aggregation prone (61) and called for a chaperone mechanism capable of using ATP to convert stable misfolded proteins into stable
Misfolded polypeptide: a biologically inactive, potentially toxic, monomeric polypeptide enriched in nonnative intramolecular $\beta$-sheets, which may further oligomerize to form an aggregate 
native proteins, which is very different from the mere prevention of aggregation by passive in-cage sequestration (62).

\section{Chaperones Can Unfold Misfolded Polypeptides}

In their seminal in vitro chaperone assay, Lorimer and colleagues (27) found that GroEL + GroES + ATP remained ineffective at refolding polypeptides that had already aggregated before chaperone addition. This led to the general belief that all molecular chaperones should, by definition, be able to prevent the aggregation of unfolded polypeptides, a misconception that was not corrected in 1998, when the first disaggregating chaperones were discovered (63). Chaperone activity was consequently often misleadingly described as a holdases $(64,65)$. This term is nonsense, as a chaperone may either be an enzyme (deserving the suffix "ase" by virtue of its ability to bind, convert, and dissociate a substrate as a low-affinity product of the enzymatic reaction) or may serve as a passive stoichiometric holding buffer (deserving the suffix "ing"), the present continuous form reflecting the lack of polypeptide dissociation in the long term. Strangely, the remarkable ability of GroEL + GroES or DnaK + DnaJ + GrpE to use ATP to drive the native refolding of inactive polypeptides was generally not underscored in the literature as being the chaperone activity par excellence. Rather, the more ordinary ability by most chaperones to prevent polypeptide aggregation was retained as the main functional characteristic $(64,66,67)$.

The GroEL/CCT chaperones are unfoldases. Hydrogen-deuterium exchange and fluorescence resonance energy transfer experiments showed that a stably misfolded RuBisCO polypeptide can become forcefully unfolded upon initial binding to an apo-GroEL complex and further unfolded upon ATP binding and hydrolysis by the GroEL complex $(68,69,70)$. GroEL-mediated unfolding was also observed by following real-time fluorescence changes in thioflavin $\mathrm{T}$ binding to misfolded $\beta$-sheets (61), which preceded the resumption of enzymatic activity of the substrates, and was confirmed by following the changes in the relative sensitivity to trypsin of various compact misfolded, extended unfolded, and compact natively refolded conformers of the same protein (50). Even in the absence of ATP, the unfolding of stable freeze-thawed misfolded rhodanese (FTrho) and freeze-thawed misfolded luciferase (FTluc) was observed upon mere binding, respectively to the GroEL and CCT chaperone complexes (50). Because this spontaneous ATP-independent catalytic binding-unfolding-release-refolding cycle by GroEL (Figure $2 a$ ) was soon to become inhibited by oversticky intermediates that failed to dissociate, ATP and GroES binding was found to be indispensible for the transient reduction of the affinity of the chaperone for the oversticky inhibitory intermediates and release the latter to refold in solution to the native state (Figure $2 \boldsymbol{b}$ ).

The Hsp70/Hsp40 chaperones are unfoldases. Similarly, without ATP, bacterial eukaryotic Hsp70s, assisted by their J-domain cochaperones, can spontaneously bind misfolding polypeptides and mildly prevent their aggregation (71). Remarkably, prevention-of-aggregation activity by the Hsp70s and by Hsp110 could be greatly increased in the presence of ATP. This suggested that there are two complementing modes of Hsp70 binding: (a) The first is a low-affinity binding of apo- or ATP-bound Hsp70 to bulky misfolded polypeptides with exposed hydrophobic residues; and $(b)$ the second is a high-affinity binding (also called locking) of the ADP-bound chaperone to an extended polypeptide at a stretch of hydrophobic residues (72). The chaperone transiently entraps the locally unfolded polypeptide in a state that is poised, upon release, to refold to the native state (Figure 3) (73, 74).

Moreover, bacterial Hsp70 (DnaK), assisted by the cochaperone Hsp40 (DnaJ), was shown to need the energy of ATP hydrolysis to forcefully unfold metastable FTluc monomeric species, 
a Catalytic unfoldase: spontaneous dissociation

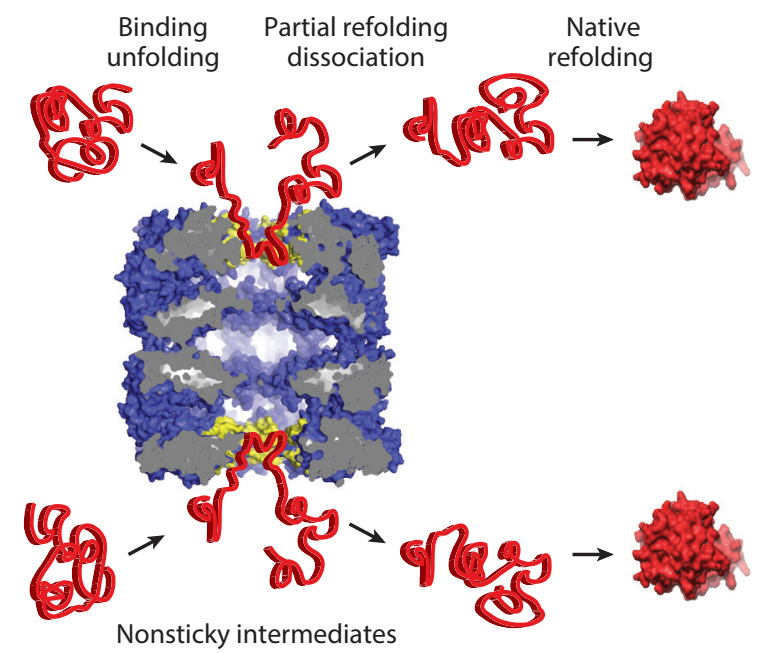

b ATP-fueled unfoldase: forceful dissociation

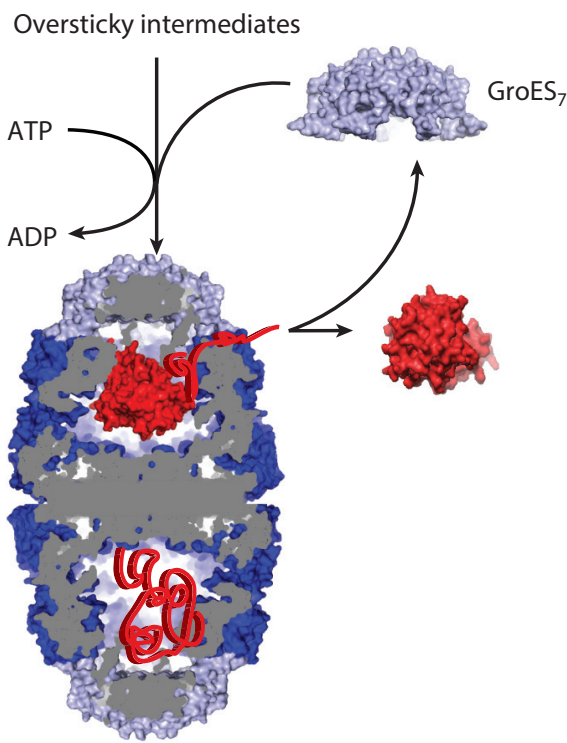

Figure 2

GroEL has two complementary types of polypeptide unfolding mechanisms. (a) Spontaneous catalytic unfolding of stable misfolded polypeptides with exposed hydrophobic surfaces. The substrate transiently binds and unfolds on the hydrophobic surfaces of the upper inner rim of the two GroEL 7 rings. Low-affinity polypeptide intermediates may then readily dissociate in the external solution, where they can freely probe various partially extended conformations on their way to the native state. (b) ATP-fueled unfolding and forceful eviction of oversticky unfolded intermediates, which block the hydrophobic catalytic unfoldase surfaces of the GroEL $\mathrm{L}_{7}$ rings. The transient binding of $\mathrm{GroES}_{7}$ mobile loops and ATP hydrolysis may then transiently decrease the hydrophobic exposure of the GroEL-binding sites, causing the dissociation of the oversticky polypeptides alternatively in the two chambers of the chaperone complex, thereby working as a two-stroke unfolding motor and allowing the polypeptides to refold to the native state both in the cavity and in the external solution. 


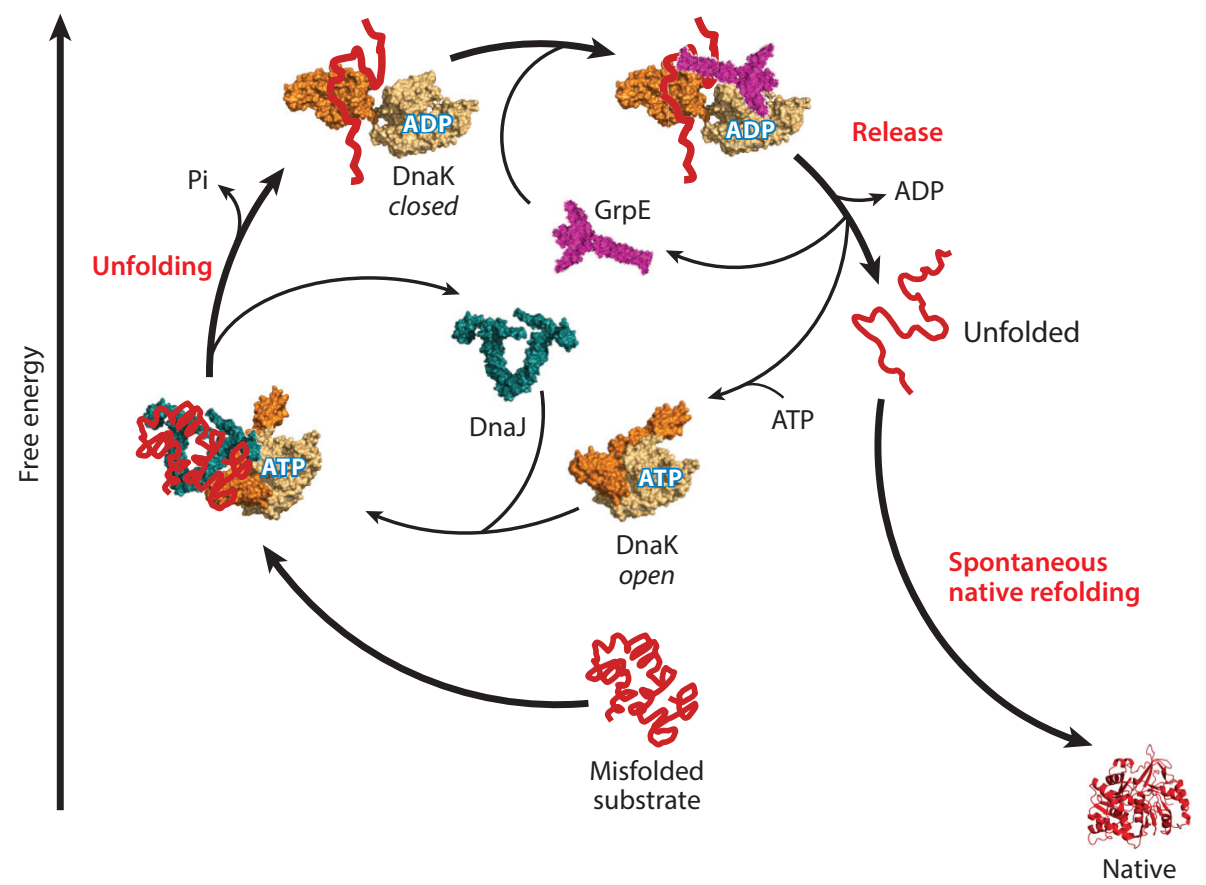

Figure 3

Hsp70 is an ATP-fueled polypeptide unfoldase. The cochaperone DnaJ (Hsp40) targets an inactive misfolded polypeptide to an opened DnaK (Hsp70) ATP. Binding triggers ATP hydrolysis and the fastening of the DnaK protein-binding domain onto the bound bulky segment of the misfolded polypeptide substrate. When successful, this may cause the unfolding of the bulky misfolded substrate and the locking of the protein-binding domain of the DnaK ADP around the newly unfolded polypeptide intermediate. Upon GrpE-accelerated ADP release, the unfolded intermediate is released in solution, where it can spontaneously refold freely into a low-affinity, natively refolded product of the unfoldase reaction. Abbreviation: $\mathrm{Pi}$, orthophosphate.

which upon subsequent GrpE-mediated dissociation could refold into native luciferase even in the absence of ATP (Figure 3) (73). Unfolding was independently demonstrated by a transient loss of thioflavin T binding to the FTluc and by the transient increase of sensitivity of the FTluc to limited trypsin digestions. A similar ATP-dependent unfolding, leading subsequently to spontaneous native refolding, was later demonstrated in the cases of human cytoplasmic Hsp70 + Hsp40 and Hsp110 + Hsp40 (74, 75).

Although all J-domain proteins likely act as cochaperones of the Hsp70 or Hsp110 unfolding chaperones, not all necessarily bind directly to the polypeptide substrates. For example, the PAM16/18 J-proteins, which are anchored at the mitochondrial membrane, participate in the targeting of ATP-bound mortalin (Hsp70) to the protein import pores. Upon ATP hydrolysis and mortalin locking on an entering polypeptide, the mitochondrial chaperone dissociates away from the pore and thus also from its J-anchors to entropically pull and unfold the locked-upon translocating polypeptide (76). Similarly, Sec63 is a J-protein at the membrane of the endoplasmic reticulum that participates in the binding of ATP-bound BIP (Hsp70) at the protein import pore complex (77). Upon ATP hydrolysis and the locking on an entering cytoplasmic polypeptide, BIP dissociates away from the pore and thus also away from the Sec63 J-protein to entropically pull and unfold the locked-upon translocating polypeptide into the endoplasmic reticulum lumen 
(78). Thus, although some J-proteins, in particular the Hsp40s, can bind misfolded polypeptide substrates, they do not necessarily cause protein unfolding (73); however, partial unfolding has also been observed in some cases (79). Recently, a DnaK-, DnaJ-, and ATP-dependent expansion action has been independently demonstrated by single-molecule spectroscopy (80).

The small heat-shock protein chaperones are probably unfoldases. The small heat-shock proteins (sHsps) have a conserved crystalline domain. In plants, they are predominantly induced by abiotic stress, and mutations in human sHsps cause cataract and degenerative diseases (81). Like all sHsps, human $\alpha \mathrm{B}$-crystalline can tightly bind and prevent the aggregation of artificially unfolded polypeptides or of stress-denaturing polypeptides. The sHsps have highly dynamic quaternary structures, which may be key to their unique ATP-independent mode of action (82-86). Thermodynamic considerations predict that mere binding of the misfolding polypeptides could reduce the highly dynamic exchange between the subunits of the sHsp oligomers, which could be balanced by a transient increase in the free energy of the bound misfolded substrates, i.e., in their partial unfolding. This implies that upon dissociation the polypeptides may spontaneously fold to the native state. When dissociation is too slow, the eviction of oversticky intermediates may require further assistance from ATP-consuming unfolding chaperones, such as Hsp70/Hsp40 and GroEL/GroES, to dissociate and finally refold (Figure 4) (85, 87, 88).

The Hsp90 chaperones are probably unfoldases. Hsp90 is present in prokaryotes and in all ATP-containing compartments of eukaryotic cells. In human cell cultures, Hsp90 is the most abundant protein and can reach up to $2 \%$ of the total protein mass of cancer cells. It forms dimers, which can spontaneously bind to misfolding polypeptides, predominantly at the inner surfaces of the N-terminal domains (89), thereby preventing the substrate aggregation without ATP (90). Hsp90s can undergo dramatic ATP-dependent structural rearrangements under the control of various eukaryotic cochaperones $(91,92)$. In the ATP- and ADP-bound state, the two protein-binding surfaces that are on the surface of the $\mathrm{N}$-terminal domains in the Hsp90 dimer are closely facing each other. Upon ADP release, the surfaces become drawn apart and twist away from one another, allowing a possible stretching by torsion motion that could locally unfold misfolded structures in a chaperone-bound polypeptide substrate (Figure 5). Zylicz and colleagues (93) showed that Hsp90 binding can partially unfold the transcription factor p53 and that ATP hydrolysis may subsequently cause the dissociation of the p53-Hsp90 complex, allowing refolding of $\mathrm{p} 53$ into an active conformation that can bind DNA promoter sequences.

\section{Chaperones Can Disaggregate Large Insoluble Aggregates}

All of the above experiments focused on chaperone substrates that were either unfolded or misfolded monomeric species that did not yet aggregate. A pivotal finding nearly two decades ago (63, 97) was that Hsp70 (DnaK) alone, but more efficiently in collaboration with Hsp40 (DnaJ) and Hsp104 (ClpB), can use the energy of ATP hydrolysis to forcefully solubilize and reactivate misfolded polypeptides that were already aggregated into stable inactive oligomers prior to chaperone addition.

A first indication that, in contrast to the GroEL system, the DnaK/Hsp70 chaperone system can act on already inactive oligomeric aggregates was presented by Hartl and collaborators (71). When luciferase was first heat-inactivated without chaperones and then supplemented with DnaK, DnaJ GrpE, and ATP, a minor fraction was refolded to the native state in a strict ATP-dependent manner (71). In 1995, Zylicz and colleagues (94) used gel filtration to further demonstrate that Hsc70 can act on polypeptides that are already aggregated in the form of stable soluble oligomers, i.e., that 


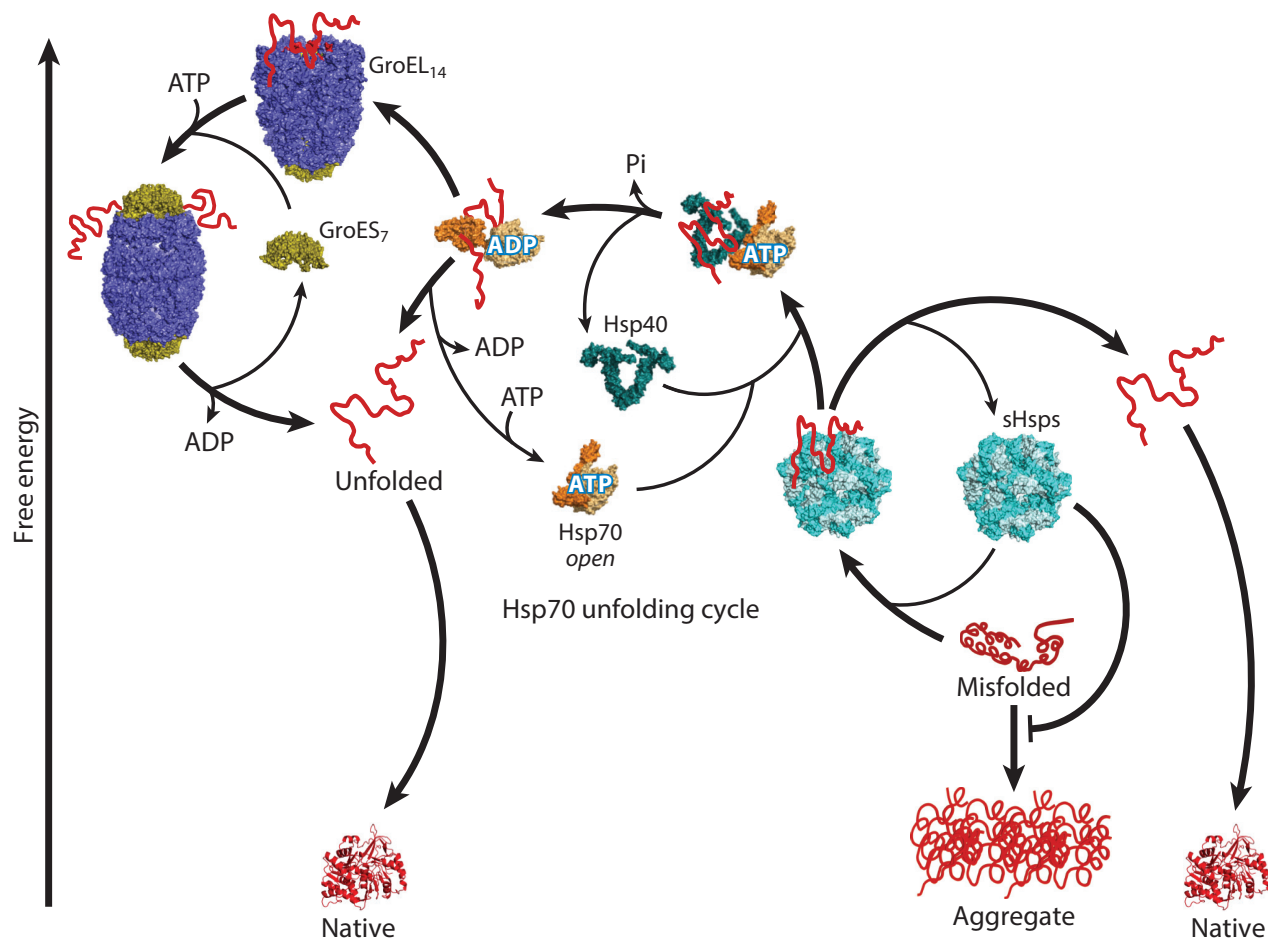

Figure 4

Small heat-shock proteins (sHsps) may have two complementary types of polypeptide unfolding mechanisms. The first is spontaneous unfolding of stable misfolded polypeptides with exposed hydrophobic surfaces that transiently bind and partially unfold on the hydrophobic surfaces on the sHsp oligomers. Low-affinity polypeptide intermediates may then readily dissociate in solution, where they can freely probe various partially extended conformations on their way to the native state (right cycle). The second is ATP-fueled unfolding and eviction of oversticky unfolded intermediates from the unfolding surfaces of the sHsp oligomers by Hsp70/Hsp40, leading directly to the native state (middle cycle). ATP-fueled unfolding by GroEL/GroES may also be needed to reach the native state (left cycle). Abbreviation: Pi, orthophosphate.

chaperones can act as ATP-fueled disaggregating chaperones. Thus, following addition of Hsc70 and ATP, heat-denatured RNA polymerases, which migrated on gel filtration as large soluble inactive oligomers, were converted into active low-molecular-weight enzymes. This was confirmed with heat-denatured glucose-6-phosphate dehydrogenases that migrated on gel filtration as soluble inactive oligomers. Following treatment with excess DnaK, DnaJ, GrpE, and ATP, the heatdenatured substrates turned into active enzymes at the cost of about 300 ATPs per refolded glucose-6-phosphate dehydrogenase (95). Thus, chaperone-mediated repair of a misfolded species was apparently 10-100 times less expensive in terms of ATP consumption, than the alternative of having to degrade and resynthesize a new protein (73).

The Hsp100/ClpB-Hsp70/DnaK are unfoldases/disaggregases. Similarly, heat preaggregated malate dehydrogenase or luciferase, which scattered light and/or migrated on gel filtration as large stable inactive oligomers, was found to be efficiently deoligomerized and reactivated by the yeast chaperone system Hsp70, Hsp40, and Hsp104 (63), or the orthologous E. coli chaperones DnaK, DnaJ, GrpE, and ClpB, in a strict ATP-dependent manner $(96,97)$. Bacterial ClpB and its 


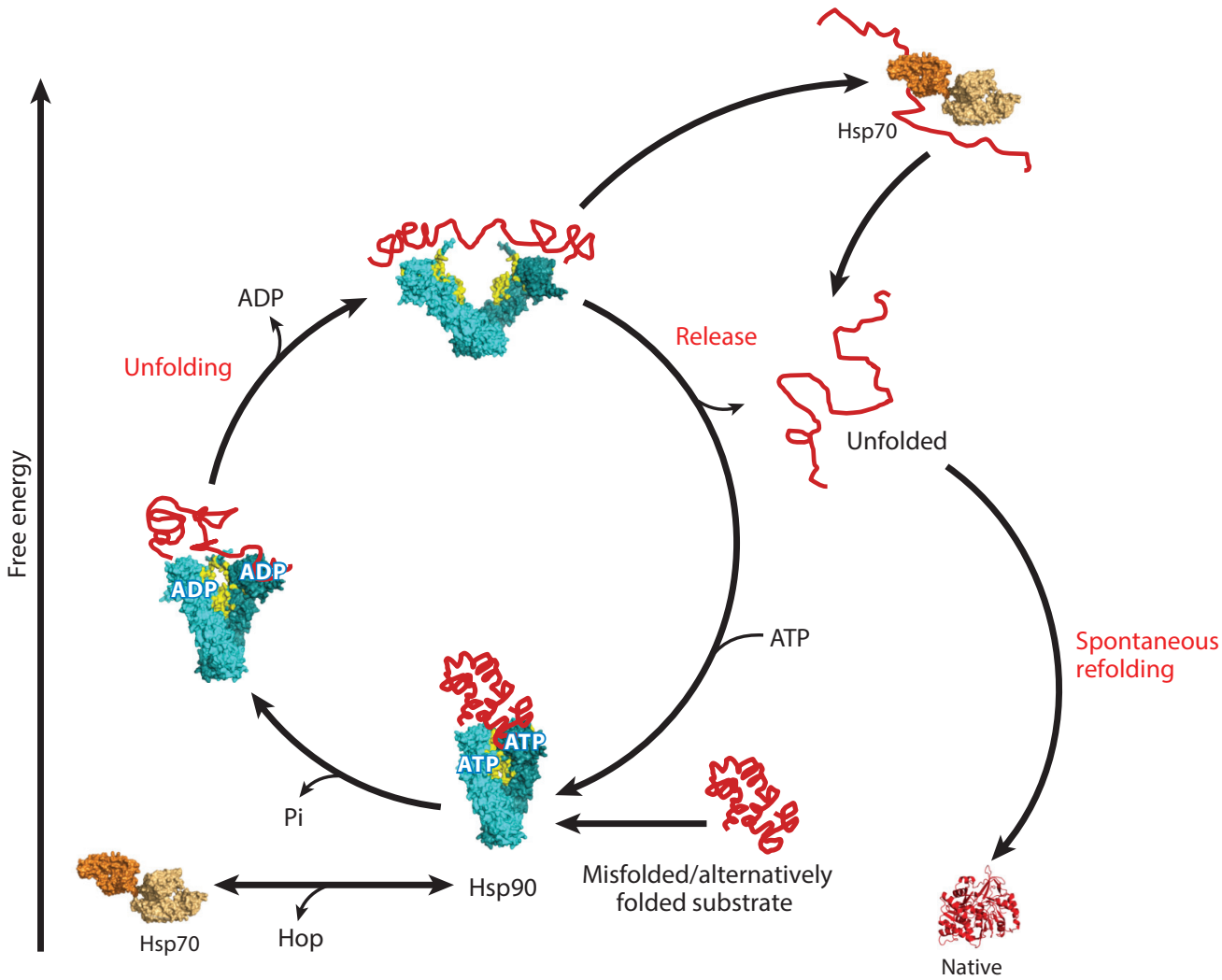

Figure 5

Hypothetical cycle for Hsp90 acting as a polypeptide unfolding enzyme. Alternatively folded or partially misfolded polypeptides (red) can directly bind or be handed by Hsp70 to ATP-bound Hsp90 dimers, where they may bind to the hydrophobic inner surfaces (yellow) principally on the nucleotide-binding domains (138). In the Hsp90 dimer, ATP hydrolysis and ADP dissociation cause structural changes that increase the distance between the two nucleotide-binding domains. This may apply an unfolding force on the bound polypeptide substrates. Upon dissociation, the partially unfolded intermediate may become further unfolded by Hsp70 and the J-domain cochaperones or directly refold to the native state. Abbreviations: Hop, Hsp70-Hsp90 organizing protein;

$\mathrm{Pi}$, orthophosphate.

eukaryotic ortholog Hsp104 are ring-shaped hexamers, which upon hydrolyzing ATP can apply a peristaltic-like unfolding force by power strokes on misfolded polypeptides interacting on the $\mathrm{N}$-terminal side of the ring. In turn, the polypeptides may become partially or completely threaded through the central channel of the chaperone cylinder (98). DnaK has a dual role, both upstream as an activator of the disaggregating chaperone $\mathrm{ClpB}(99,100)$ and downstream as a possible ATP-fueled unfolding machine that can complete the unfolding of polypeptides that were only partially disentangled by ClpB (97). Remarkably, sequence-wise, and structurally, ClpA is highly homologous to $\mathrm{ClpB}$. Yet, ClpA and $\mathrm{ClpX}$ are both cylindrical unfolding enzymes that form a specific complex with the $\mathrm{ClpP}$ protease, which is a heptameric cylinder, and that together actively unfold and degrade misfolded proteins in an ATP-dependent manner, similar to the eukaryotic proteasome $(98,101)$. By contrast, $\mathrm{ClpB}$ is an unfolding enzyme that does not associate with ClpP and thus cannot degrade misfolded proteins but rather acts in concert with DnaK to disaggregate, unfold, and refold them into native proteins (Figure 6) (102, 103). This mechanism was predicted by Rothman \& Kornberg (1), who suggested as early as 1986 that cells should have unfolding 


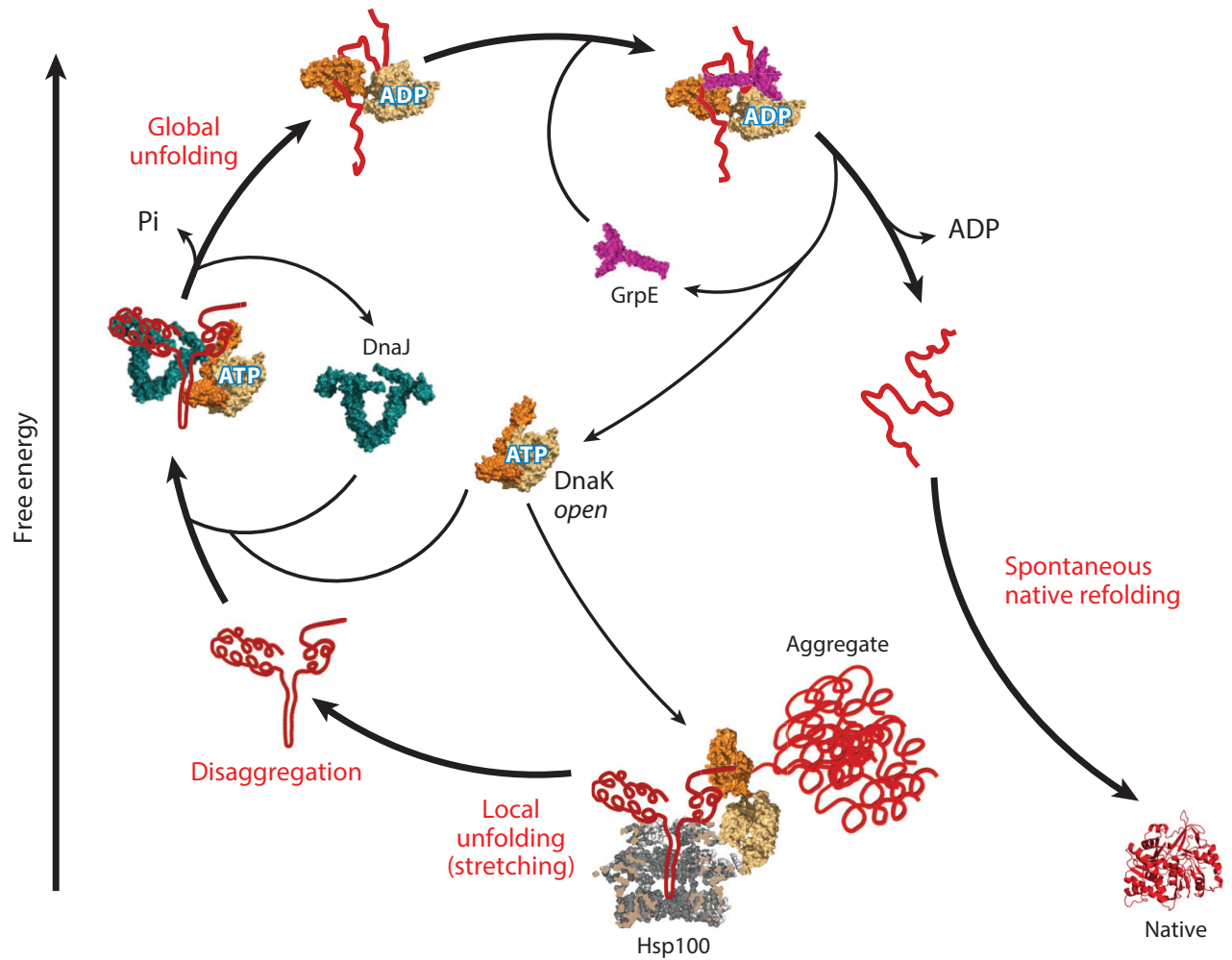

Figure 6

Hsp100/ClpB is a disaggregating machine. Hsp100/ClpB forms an ATP-fueled disaggregating cylindrical hexamer controlled by a regulatory $\mathrm{M}$ domain. The $\mathrm{N}$-terminal side of the Hsp100/ClpB cylinder can bind exposed misfolded polypeptide loops on the surface of aggregates. Hsp70/DnaK concomitant binding to the aggregates and to the $\mathrm{ClpB} M$ domain activates ATP hydrolysis, fueling power-stroke movements in the $\mathrm{ClpB}$ cavity, which can stretch bound polypeptide loops and disentangle a misfolded polypeptide from the aggregate. Once released from the Hsp100/ClpB cavity on either side, the partially misfolded soluble polypeptide may be further forcefully unfolded by the ATP-fueled action of the DnaK/DnaJ/GrpE machinery and, once released in solution, spontaneously refold to the native state. Abbreviation:

$\mathrm{Pi}$, orthophosphate.

enzymes associated to proteases, as it turned out to be the case of $\mathrm{ClpX}$ and $\mathrm{ClpA}$ associated to ClpP. They further predicted that evolution might have selected unfolding enzymes that lost their proteolytic activity, as it turned out to be the case of $\mathrm{ClpB}$, and that would still recognize incorrectly folded proteins but provide them with a renewed chance to fold properly $(96,97)$. Today, all chaperones that carry typical Walker A and Walker B motifs, whether associated to proteases or not, are thought to act as cylindrical unfolding nanomachines. In their central pore, an ATP-fueled power stroke, initiated by the protruding pore loop of one subunit, results in synergic movements in all six loops, thereby coordinately gripping and applying an unfolding/pulling force onto single misfolded chains or polypeptide loops exposed on the aggregate surface (104).

The Hsp70/Hsp110s are unfoldases and disaggregases. In the cytoplasm and the endoplasmic reticulum of animal cells, which apparently do not have Hsp100/ClpB orthologs, there is 
a

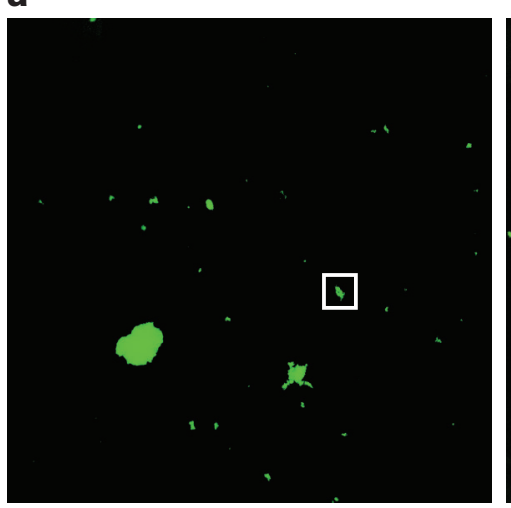

$\overline{50 \mu \mathrm{m}} \quad$ Intact

b

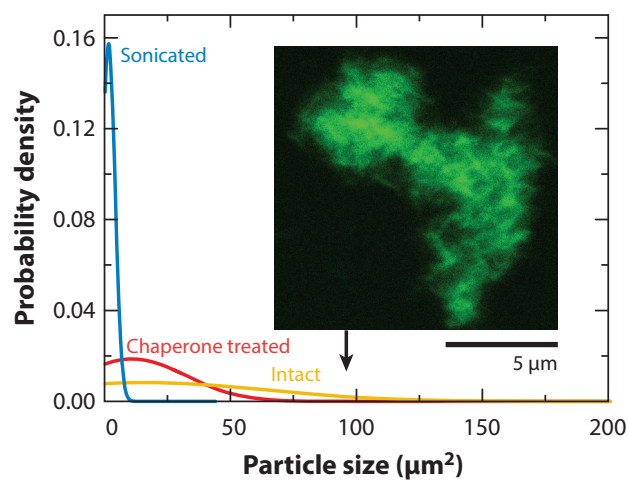

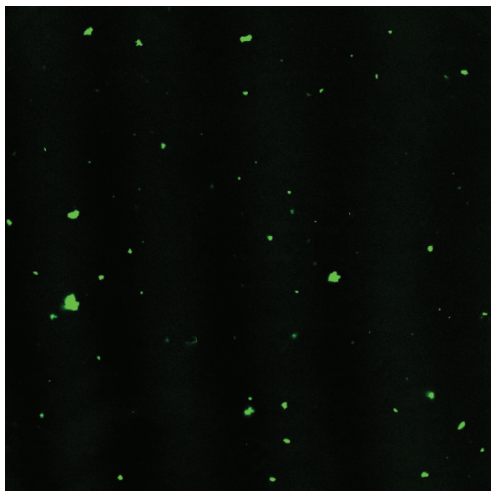

Chaperone treated

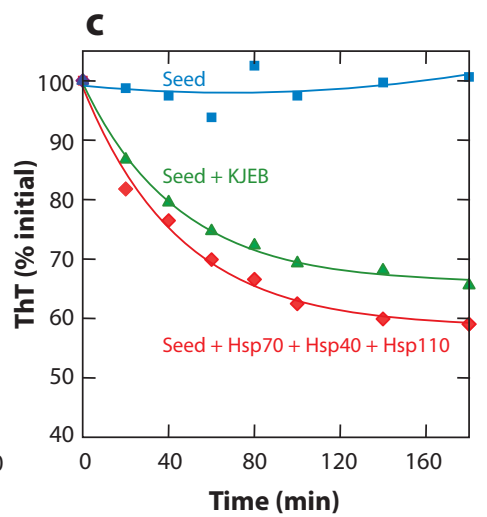

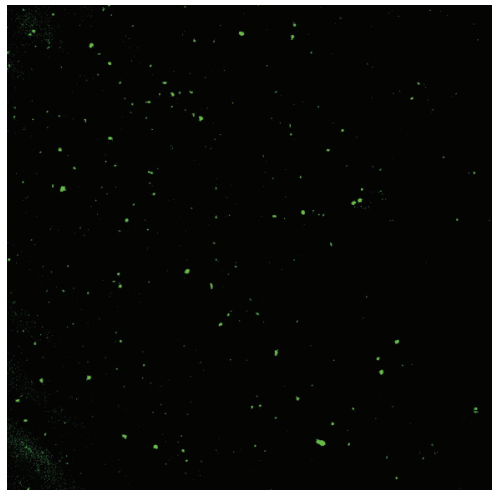

Sonicated

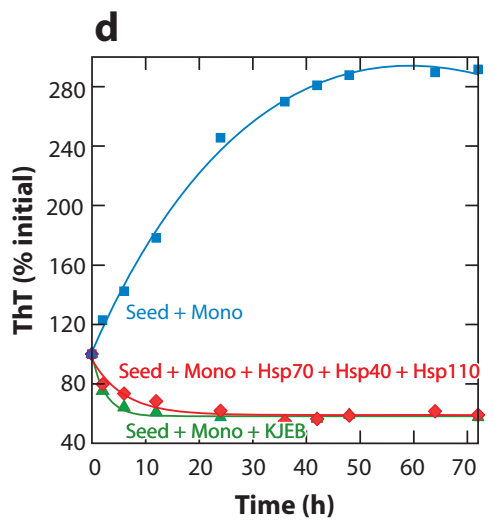

Figure 7

Human Hsp70/Hsp110 efficiently break apart stable $\alpha$-synuclein fibrils. (a) (left) Confocal microscopy of mature $\alpha$-synuclein fibrils ( $5 \mu \mathrm{M}$ of protomer equivalents) stained with thioflavin T (ThT) before or (middle) after a 3-h treatment with $8 \mu \mathrm{M}$ Hsp70 $+8 \mu \mathrm{M}$ Hsp110 + $6 \mu \mathrm{M}$ Hsp40 and ATP or (right) after 32 cycles of sonication. (b) Distributions of particle sizes before and following chaperone or sonication treatments as in panel $a$. (Inset) A high-resolution example of intact fibrils before treatment. $(c)$ Timedependent changes in ThT fluorescence of sonicated $\alpha$-synuclein fibrils without (squares) or with molecular chaperones and ATP (diamonds, triangles). (d) Time-dependent changes of ThT fluorescence at $37^{\circ} \mathrm{C}$ of sonicated $\alpha$-synuclein seeds $(2.5 \mu \mathrm{M})$, which were pretreated with molecular chaperones and ATP (diamonds, triangles) or not (squares) as in panel $c$, and supplemented to $60 \mu \mathrm{M}$ $\alpha$-synuclein protomers. Abbreviation: Mono, monomeric unstructured $\alpha$-synuclein.

another efficient disaggregating chaperone machinery composed of two evolutionarily related chaperones, Hsp70 and Hsp110. Hsp110 is structurally and functionally related to the Hsp70 family. Like Hsp70, it is a bona fide ATP-fueled Hsp40-regulated unfolding chaperone by itself (74), which is not to be confused with the AAA+ proteins Hsp104/Hsp100/ClpB that are unrelated to Hsp70. Without ATP, human Hsp110 was shown to induce the release of an unfolded inactive luciferase substrate prelocked into a human Hsp70, and reciprocally, Hsp70 was shown to induce the release of an unfolded inactive luciferase substrate prelocked into a human Hsp1 10 (74). Moreover, human Hsp110 and Hsp70 chaperones were shown to concertedly act as equal partners in a functional heterodimer, where each likely alternatively hydrolyzed ATP to disaggregate and unfold misfolded polypeptides entangled into large stable aggregates by a yet unclear mechanism 
(74, 105-108). Thus, very stable $\alpha$-synuclein fibrils can be broken apart in the test tube by an equimolar combination of human Hsp70, Hsp110, and Hsp40 in a strict ATP-dependent manner that is as efficient as the bacterial chaperones DnaK + DnaJ + GrpE + ClpB (KJEB) and nearly as efficient as the mechanical action of sonication (Figure $7 a, b$ ). When applied to sonicated $\alpha$-synuclein fibrils, Hsp110, Hsp70 + Hsp40, and ATP could significantly reduce the amount of wrong $\beta$-sheets that specifically bound thioflavin $T$, in contrast to the intrinsically unfolded monomeric $\alpha$-synuclein that lacks structures and therefore did not bind thioflavin $\mathrm{T}$ (Figure $7 c$ ). Moreover, once chaperone treated, the sonicated $\alpha$-synuclein fibrils lost their ability to seed the spontaneous formation of insoluble $\alpha$-synuclein fibrils from a large molar excess of monomeric $\alpha$-synuclein (Figure $7 \boldsymbol{d}$ ). This illustrates the importance of the cytosolic Hsp70/Hsp110 disaggregating machinery in preventing and averting the formation of early misfolded and aggregated conformers in human cells. Such conformers are extremely dangerous, as they can further seed and accelerate the formation of toxic protofibrils from cytoplasmic proteins, such as $\alpha$-synuclein and tau, causing synucleinopathies (109) and tauopathies (110), respectively.

Interestingly, the cytoplasm of plants, yeast, and fungi harbors both Hsp100/ClpB-Hsp70/ DnaK and Hsp70/Hsp110 disaggregating machineries, suggesting that they do not fully overlap in terms of their specificity toward various aggregates. Thus, although the human cytoplasm and endoplasmic reticulum propitiously carry at least one effective chaperone disaggregation system (Hsp70/Hsp110), unlike yeast and plants, they may suffer from a lack of the complementary Hsp104/Hsp70-based disaggregating machinery, and this loss of function likely contributes to the high sensitivity of aging mammalian cells to toxic protein aggregates.

\section{Chaperones Can Catalytically Unfold Stable Misfolded Polypeptides}

Different molecular chaperones have been recently found to act as iterative unfolding catalysts. They can apparently convert a majority of metastable misfolded polypeptide substrates into sufficiently unfolded, low-affinity polypeptide products. Upon release, these can spontaneously refold into low-affinity native proteins, acting as final functional products of the chaperone reaction. Catalysis was first shown to be the case of bacterial Hsp70 (DnaK), where a single molecule of DnaK (in the presence of ATP and substoichiometric amounts of DnaJ and GrpE) could processively convert several molecules of inactive FTluc monomers into native luciferase. This reaction could occur in about five times as many iterative cycles of binding, unfolding, release, and spontaneous refolding, thereby rehabilitating one luciferase molecule at the cost of five hydrolyzed ATPs (Figure 3). This low energy cost was estimated to be at least a thousandfold lower than for alternative controlled degradation of a misfolded luciferase polypeptide by ATP-fueled proteases, followed by its resynthesis into a new functional protein (73). A similar catalytic unfolding mechanism with a higher ATP cost was demonstrated also in the case of eukaryotic Hsp70 and Hsp110 (74). In addition, catalytic unfolding was demonstrated in the case of GroEL and CCT chaperones, which were found to efficiently convert stable misfolded freeze-thawed rhodanese polypeptides into native rhodanese enzymes in iterative cycles of binding, unfolding, release, and spontaneous native refolding that did not require ATP (50). ATP hydrolysis was necessary only to transiently reduce the high affinity of particular intermediates that were excessively sticky and consequently failed to dissociate and refold to the native state within a biologically relevant timescale (Figure 2). It is tempting to speculate that the ability to drive the extension or the decompaction of stably misfolded, aggregated, or alternatively folded proteins into partially unfolded, natively refoldable, or protease-degradable proteins is the common functional denominator for the main families of molecular chaperones (62). If this was the case, then the field should reconsider term chaperone because it implies only a passive binding function for these proteins. 


\section{CHAPERONE ACTIVITY DOES NOT DEPEND ON IN-CAGE CONFINEMENT OF FOLDING INTERMEDIATES}

In 2011, Hartl and Horwich received the Albert Lasker Award for Basic Medical Research for their discoveries concerning the cell's protein-folding machinery, exemplified by cage-like structures that convert newly made proteins into their biologically active forms. It was further specified in the award syllabus that "Hsp60 forms a barrel that grabs the sticky patches of the unfolded protein, moves it into the barrel, and closes a lid on top of it. In isolation, the protein can now try out alternate conformations, giving it a chance to fold into its correct structure, without bumping into other proteins" (see http://www.laskerfoundation.org/awards/show/chaperone-assisted-proteinfolding/).

Although a mechanism of transient confinement within the closed chamber of GroEL or CCT, previously dubbed the Anfinsen cage by Ellis (53), may be optimal for some particular polypeptides smaller than $55 \mathrm{kDa}$, transient in-cage confinement is not an obligate condition associated with the mechanism of molecular chaperones in general. Hence, following the ATPfueled unfolding of misfolded FTluc species by DnaK, which is a $70-\mathrm{kDa}$ chaperone that does not form cage-like oligomeric structures, over $95 \%$ of the DnaK-released unfolded luciferase species reached their native state while they were completely free in solution (73). It is thus unwarranted to assume that all chaperone-unfolded polypeptides released in solution would have to obligatorily aggregate. It is therefore unnecessary to assume that all the chaperones must act by way of preventing protein aggregation. By contrast, most chaperones now appear to act as nanomachines that can unfold misfolded or alternatively folded polypeptide intermediates and also, on occasion, prevent their aggregation (also called holding), especially during heat shock when releasing thermolabile polypeptides in solution would fruitlessly result in the accumulation of increasingly stable chaperone-resistant aggregates (reviewed in 52, 65). In other words, prevention of aggregation is not an obligate part of the chaperone unfolding mechanisms, although it may often be a collateral advantage of chaperone activities.

The social adage "this is an exception that proves the rule" does not apply to protein science, in which pettier exceptions should have the power to invalidate long-accepted molecular mechanisms. Hence, when unfolded $90-\mathrm{kDa}$ aconitase was used as a substrate, GroEL effectively refolded it to the native state in a strict GroES- and ATP-dependent manner, although only half of it could physically fit within the chaperone cavity and GroES could not physically cap it. Horwich and collaborators suggested that following GroEL binding and the ATP-mediated GroES binding, aconitase should be released in the outside solution, where it should complete its folding to native state, without aggregating, as expected by the in-cage model $(57,111)$. If not to become sequestered and prevented from aggregating when outside the cavity, what could have been the ATP-dependent processes that aconitase had to undergo while being bound to the upper rim of the GroEL cavity? Based on recent data, the most likely answer is: unfolding $(50,69)$.

Even in the case of the 30-kDa rhodanese polypeptide, which can be compelled to refold within the chaperone cage under a sealed GroES cap [in the case of the so-called SR1 mutants that do not dissociate GroES (112)], this may be much less effective than when native rhodanese folding is allowed to take place unrestrictedly outside the chaperone cavity. Thus, when following unfolding on the upper inner rim of the GroEL cavity, the freeze-thawed rhodanese species were released and allowed to freely probe for various extended conformations outside the cavity; they refolded seven times more efficiently than when they were compelled to do so within the cramped space of a GroEL cavity under a sealed GroES lid (50).

The so-called Anfinsen cage model is thus an elegant model (113) primarily based on the initial observation by Goloubinoff et al. (27) that the GroEL-GroES system is unable to act once 
RuBisCO aggregates were already formed. Thereafter, the chaperone field overstatedly assumed that, when left free in solution, most nascent or stress-unfolded polypeptides should irrecoverably aggregate into chaperone-resistant species. This assumption was proven wrong a decade later when the Hsp104/Hsp70 (63) and ClpB/DnaK chaperones $(96,97)$ were found to act as effective ATPfueled disaggregating/refolding nanomachines capable of converting stable preformed insoluble aggregates into native proteins.

Anfinsen's seminal observations (11) and Rothman \& Kornberg's initial suggestions (1) about the existence of cellular unfoldases point to another elegant model, which is now substantiated by three decades of chaperone research: When polypeptides partially unfold in vitro or in stressed cells, they may indeed readily misfold because completely unfolded polypeptides with large patches of water-exposed hydrophobic residues are thermodynamically unstable. The resulting stable misfolded and aggregated species may yet expose some residual characteristic hydrophobic surfaces that can be recognized by the specific hydrophobic surfaces on chaperones, such as the GroELbinding sites that face the inner upper rim of the cavity $(72,114)$. Mere binding might already exert a significant unfolding (69) or decompaction on the substrate by annealing $(89,115)$, which could suffice to lower the kinetic barrier between the metastable misfolded states and unfold the intermediates in such a way that upon release they would spontaneously reach their native state. Hence, when a stably unfolded inactive luciferase, which had been first prelocked within an ADP-DnaK complex, was released in solution by GrpE addition in the total absence of ATP, it spontaneously refolded to the native state with an efficiency approaching 100\% (73). Thus, contrary to artificial urea unfolding, chaperone-unfolded polypeptides may not obligatorily misfold and aggregate upon their release in solution. It is indeed more likely that protein evolution has favored the native structures with the lowest free energy. Yet, to fulfill more elaborate functions, protein evolution may have had to compromise with stability and allow higher risks of misfolding on the way to the native state (116), especially in the case of eukaryotic multidomain polypeptides (66). To achieve the native state, these proteins may have had to develop a strong dependence on chemical chaperones (117) and on protein chaperones, acting as polypeptide unfoldases, which would have prevented the formation of inactive, potentially toxic aggregates and provided already damaged species with a renewed chance to spontaneously regain their native folding and to avoid degradation and being resynthesized at a very high ATP cost. Upon binding and unfolding very early misfolding intermediates on the aggregation pathway, the various polypeptide unfoldases could thus buffer the evolution of increasingly complex multidomain polypeptides $(116,118,119)$.

\section{MOLECULAR CHAPERONES SHOULD REVERT TO THEIR ORIGINAL NAME: UNFOLDING ENZYMES}

In 1987, Ellis levied on the scientific community a social term, chaperone, to characterize the presumed molecular function of a group of proteins suspected at the time to mainly prevent the aggregation of other proteins and, occasionally, also promote the proper assembly of functional oligomers (2). Yet, after about 30 years of research, there is still a need to find the common biochemical property of all molecular chaperones. Not all can prevent the aggregation of other proteins. Defining them as antiaggregation molecules would exclude, for example, the disaggregating $\mathrm{ClpB} / \mathrm{Hsp} 100$ machineries that do not prevent aggregation per se. By contrast, not all chaperones can refold inactive proteins to their native state, and defining them as folding molecules would exclude, for example, Hsp90, Hsp40, and trigger factor. In addition, not all molecular chaperones are Hsps, and reciprocally, not all Hsps are molecular chaperones. Defining chaperones as Hsps would exclude proteins that are not induced by heat and, moreover, erroneously assign a chaperone function to heat-induced nonchaperone proteins (120). Yet, there is now compelling 
evidence of two common properties to all chaperones: (a) They can specifically recognize and bind to misfolded, aggregated, or alternatively folded proteins, while leaving untouched the large majority of surrounding native or intrinsically unfolded proteins in the cell. (b) Upon binding and in some cases also upon hydrolyzing ATP, they can cause the local or global unfolding, decompaction, pulling, or extension of the bound polypeptides. Release of these polypeptides can then lead to native refolding or to controlled degradation in a protease chamber $(62,65)$. Recent findings clearly show that most, if not all, classes of molecular chaperones behave as polypeptide unfolding enzymes or more simply as unfoldases. This would categorize them among the class 5 isomerases. Indeed, similar to peptidyl-prolyl isomerases, the unfoldases apparently act on their bound misfolded or alternatively folded substrates to change their conformation without changing their overall chemical composition, thereby converting them into differently folded, low-affinity native polypeptide products (65). In 1986, Rothman \& Kornberg (1) suggested the term unfolding enzymes, but at the time, this lacked experimental support. Instead, the term molecular chaperone was suggested a year later (2) and continues to be used. This latter term is inspired from the social function of preventing unproductive associations among teenagers. It implies a molecular function of preventing useless protein aggregations and fails to account for the remarkable ability of most chaperones to act as polypeptide unfoldases that can edit the three-dimensional structure of proteins in the highly crowded environment of the cell (121). It would thus appear that the social term chaperone is an incomplete description and should be avoided. If any, the social term molecular police would much better correspond to their observed cellular functions (122). Instead, the original term from Rothman \& Kornberg (1) of unfolding enzymes should be reinstated, as it is scientifically more accurate and informative about the mechanism by which these proteins regulate cellular proteostasis $(123,124)$.

\section{THE UNFOLDASES CAN PROOFREAD THREE-DIMENSIONAL PROTEIN STRUCTURES AND THEREBY CONTROL PROTEOSTASIS}

By virtue of their ability to act as polypeptide unfolding enzymes that can specifically target highaffinity misfolded conformers while remaining indifferent to properly folded native proteins, the various unfoldases can verify the quality of the proteins in the crowded environment of the cell. They can proofread the three-dimensional structures of proteins; bind and unfold only those presenting signs of misfolding, such as hydrophobic surfaces; and convert them into harmless spontaneously refoldable or degradable proteins.

In the flowchart of molecular biology, intricate information needs to be faithfully transferred between various forms of complex polymers: DNA $\rightarrow$ RNA $\rightarrow$ proteins. Owing to its high complexity, the information contained therein is fragile, and the transfer is error prone. Hence, although the major function of DNA polymerase is to catalyze the forward template-dependent $5^{\prime}-3^{\prime}$ polymerization of DNA, this polymerase is equipped with a built-in detection system for errors. By virtue of its high affinity for wrongly paired nucleotides and its unique ability to conditionally act backward on them as a $3^{\prime}-5^{\prime}$ exonuclease, while remaining indifferent to properly paired nucleotides, DNA polymerase can proofread DNA, avoid nonallelic homologous recombination, and thus control the quality of the transferred information to daughter strands (for a review see 125). Proofreading quality-control mechanisms exist also for mRNA transcription (126) and mRNA translation by the ribosomes (127). Protein folding is also a very intricate and error-prone process, especially under heat shock. The various members of the unfoldase network may thus proofread the three-dimensional structures of all the proteins in the cells at a steady state, during growth and under stress. When necessary, the unfoldases can transiently arrest the spontaneous misfolding process of a protein, and by unfolding, they may even move backward by undoing the
Proteostasis: all the cellular processes controlling the life of a protein, from birth in the ribosome to folding, translocation, assembly, activation and protease-mediated degradation 
faulty structures before resuming the proper forward spontaneous folding process to the native state. When there is no stress, the unfoldases may also undertake physiological functions, such as driving vesicular trafficking and protein import into organelles, similar to police regulating city traffic instead of having to apprehend and process criminals who thrive under more stressful conditions.

\section{THE IMPORTANCE OF UNDERSTANDING POLYPEPTIDE UNFOLDASE MECHANISMS}

Understanding the precise mechanisms by which various classes of unfoldases may prevent the formation of, and/or actively avert, the accumulation of disease-causing protein conformers is fundamental to the design of unfoldase-based therapies against various generative protein conformational diseases $(128,129)$. Polypeptide unfoldases can specifically identify misfolded conformers and either send them to controlled degradation or rehabilitate them into functional proteins to further sustain cellular life during and following stress by buffering aggregation-prone mutations, by repairing stress-induced damage, and by inhibiting proapoptotic signals (130,131). Unfoldase overexpression may be beneficial to aging mammalian tissues, which are defective in detecting abiotic and cellular stresses and that insufficiently respond to various challenges. By contrast when increasing the unfoldase load, Hsp70 in particular might correspondingly increase the survival of cancer cells by blocking apoptosis during and following radio-, chemo-, and thermotherapies (3). It is important to understand the molecular mechanisms by which the various polypeptide unfoldases act on different toxic and alternative protein conformers to develop effective overexpressing approaches to combat degenerative protein conformational diseases and to identify specific unfoldase inhibitors to increase the sensitivity of cancer cells to aggressive treatments $(132,133)$. Moreover, in the context of global warming, it is essential to understand how in sessile organisms, such as plants that cannot escape environmental stresses, various classes of heat-induced unfoldases may accumulate during a mild priming rise of ambient temperature to effectively prevent subsequent protein damage by upcoming noxious higher temperatures typically occurring at noon. Once the heat stress is over, the unfoldases may thus rehabilitate inactive misfolded, potentially toxic conformers by unfolding them into natively refoldable harmless functional proteins $(131,134-136)$.

\section{FUTURE ISSUES}

1. Given that diverse chaperone families act as polypeptide unfoldases, what is the specificity of each unfoldase regarding various alternative and toxic misfolded polypeptide substrates in the cell?

2. What are the unfolding mechanisms and affinity profiles of the various unfoldases for misfolded, aggregated, natively unfolded and/or alternatively folded protein substrates?

3. What are the precise expression profiles and cellular quantities of chaperones/unfoldases at steady state, in development, in aging, and in response to various stresses?

4. What are the expression profiles of chaperones/unfoldases in pathologies, such as cancer and misfolding diseases?

5. Which drugs can control, and by what cellular mechanisms, the expression of chaperones/ unfoldases in pathologies and aging? 


\section{DISCLOSURE STATEMENT}

The authors are not aware of any affiliations, memberships, funding, or financial holdings that might be perceived as affecting the objectivity of this review.

\section{ACKNOWLEDGMENTS}

We apologize to all investigators whose research could not be appropriately cited owing to space limitations. Research was financed by the Swiss National Science Foundation grants 125502/1 and 140512/1. We thank Matthias Mayer, Maya Schuldiner, Edward Farmer, and Jacques Beckmann for comments on the manuscript.

\section{LITERATURE CITED}

1. Rothman JE, Kornberg RD. 1986. Cell biology: an unfolding story of protein translocation. Nature 322:209-10

2. Ellis J. 1987. Proteins as molecular chaperones. Nature 328:378-79

3. Finka A, Goloubinoff P. 2013. Proteomic data from human cell cultures refine mechanisms of chaperonemediated protein homeostasis. Cell Stress Chaperones 18:591-605

4. Finka A, Sood V, Quadroni M, De Los Rios P, Goloubinoff P. 2015. Quantitative proteomics of heattreated human cells show an across-the-board mild depletion of housekeeping proteins to massively accumulate few Hsps. Cell Stress Chaperones 20:605-20

5. Crick F. 1970. Central dogma of molecular biology. Nature 227:561-63

6. Cressey D. 2015. DNA-repair sleuths win chemistry Nobel. Nature 526:307

7. Hershko A, Ciechanover A, Heller H, Haas AL, Rose IA. 1980. Proposed role of ATP in protein breakdown: conjugation of protein with multiple chains of the polypeptide of ATP-dependent proteolysis. PNAS 77:1783-86

8. Bukau B, Weissman J, Horwich A. 2006. Molecular chaperones and protein quality control. Cell 125:44351

9. Hinault MP, Ben-Zvi A, Goloubinoff P. 2006. Chaperones and proteases: cellular fold-controlling factors of proteins in neurodegenerative diseases and aging. 7. Mol. Neurosci. 30:249-65

10. Anfinsen CB, Sela M, Cooke JP. 1962. Reversible reduction of disulfide bonds in polyalanyl ribonuclease. 7. Biol. Chem. 237:1825-31

11. Epstein CJ, Goldberger RF, Anfinsen CB. 1963. Genetic control of tertiary protein structure-studies with model systems. Cold Spring Harb. Symp. 28:439-49

12. Milo R. 2013. What is the total number of protein molecules per cell volume? A call to rethink some published values. BioEssays 35:1050-55

13. Hartl FU, Martin J, Neupert W. 1992. Protein folding in the cell: the role of molecular chaperones Hsp70 and Hsp60. Annu. Rev. Biophys. Biomol. Struct. 21:293-322

14. Ellis RJ. 2001. Macromolecular crowding: obvious but underappreciated. Trends Biochem. Sci. 26:597-604

15. Sternberg N. 1973. Properties of a mutant of Escherichia coli defective in bacteriophage $\lambda$ head formation (groE): II. The propagation of phage $\lambda$. . . Mol. Biol. 76:25-44

16. Yochem J, Uchida H, Sunshine M, Saito H, Georgopoulos CP, Feiss M. 1978. Genetic analysis of two genes, dnaf and dnaK, necessary for Escherichia coli and bacteriophage lambda DNA replication. Mol. Gen. Genet. 164:9-14

17. Tilly K, Murialdo H, Georgopoulos C. 1981. Identification of a second Escherichia coli groE gene whose product is necessary for bacteriophage morphogenesis. PNAS 78:1629-33

18. Hohn T, Hohn B, Engel A, Wurtz M, Smith PR. 1979. Isolation and characterization of the host protein groE involved in bacteriophage lambda assembly. F. Mol. Biol. 129:359-73

19. Barraclough R, Ellis RJ. 1980. Protein synthesis in chloroplasts. IX. Assembly of newly synthesized large subunits into ribulose bisphosphate carboxylase in isolated intact pea chloroplasts. Biochim. Biophys. Acta 608:19-31
(1) Suggested that cellular unfolding enzymes exist to assist protein translocation and folding and prevent aggregation.

(19) Provided evidence that a nascent polypeptide may associate with another protein before assembly into a mature oligomer. 
(27) Provided experimental evidence that a chaperone can prevent aggregation and use ATP to mediate native protein refolding.

(41) Reported sequence homology evidence that a chaperone can mediate the assembly of protein oligomers.

(42) Provided evidence that a chaperone mediates proper folding and assembly of a foreign protein in cells.
20. Laskey RA, Honda BM, Mills AD, Finch JT. 1978. Nucleosomes are assembled by an acidic protein which binds histones and transfers them to DNA. Nature 275:416-20

21. Hemmingsen SM, Ellis RJ. 1986. Purification and properties of ribulosebisphosphate carboxylase large subunit binding protein. Plant Physiol. 80:269-76

22. LeBowitz JH, Zylicz M, Georgopoulos C, McMacken R. 1985. Initiation of DNA replication on singlestranded DNA templates catalyzed by purified replication proteins of bacteriophage $\lambda$ and Escherichia coli. PNAS 82:3988-92

23. Deleted in proof

24. Zylicz M, Georgopoulos C. 1984. Purification and properties of the Escherichia coli DnaK replication protein. 7. Biol. Chem. 259:8820-25

25. Osipiuk J, Georgopoulos C, Zylicz M. 1993. Initiation of lambda DNA replication. The Escherichia coli small heat shock proteins, DnaJ and GrpE, increase DnaK's affinity for the lambda P protein. 7. Biol. Chem. 268:4821-27

26. Georgopoulos CP. 1977. New bacterial gene (groPC) which affects $\lambda$ DNA replication. Mol. Gen. Genet. 151:35-39

27. Goloubinoff P, Christeller JT, Gatenby AA, Lorimer GH. 1989. Reconstitution of active dimeric ribulose bisphosphate carboxylase from an unfolded state depends on two chaperonin proteins and Mg-ATP. Nature 342:884-89

28. Tissières A, Mitchell HK, Tracy UM. 1974. Protein synthesis in salivary glands of Drosophila melanogaster: relation to chromosome puffs. F. Mol. Biol. 84:389-98

29. Finley D, Ciechanover A, Varshavsky A. 1984. Thermolability of ubiquitin-activating enzyme from the mammalian cell cycle mutant ts 85 . Cell 37:43-55

30. Pelham HRB. 1986. Speculations on the functions of the major heat-shock and glucose-regulated proteins. Cell 46:959-61

31. Lewis MJ, Pelham HRB. 1985. Involvement of ATP in the nuclear and nucleolar functions of the $70 \mathrm{kd}$ heat-shock protein. EMBO 7. 4:3137-43

32. Eilers M, Schatz G. 1986. Binding of a specific ligand inhibits import of a purified precursor protein into mitochondria. Nature 322:228-32

33. Waxman L, Goldberg AL. 1985. Protease La, the lon gene product, cleaves specific fluorogenic peptides in an ATP-dependent reaction. 7. Biol. Chem. 260:12022-28

34. Musgrove JE, Johnson RA, Ellis RJ. 1987. Dissociation of the ribulosebisphosphate-carboxylase largesubunit binding protein into dissimilar subunits. Eur. 7. Biochem. 163:529-34

35. Musgrove JE, Ellis RJ. 1986. The Rubisco large subunit binding protein. Philos. Trans. B 313:419-28

36. Picard D, Salser SJ, Yamamoto KR. 1988. A movable and regulable inactivation function within the steroid binding domain of the glucocorticoid receptor. Cell 54:1073-80

37. Lindquist S, Craig EA. 1988. The heat-shock proteins. Annu. Rev. Genet. 22:631-77

38. Bochkareva ES, Lissin NM, Girshovich AS. 1988. Transient association of newly synthesized unfolded proteins with the heat-shock GroEL protein. Nature 336:254-57

39. Deuerling E, Schulze-Specking A, Tomoyasu T, Mogk A, Bukau B. 1999. Trigger factor and DnaK cooperate in folding of newly synthesized proteins. Nature 400:693-96

40. Pushkin AV, Tsuprun VL, Solovjeva NA, Shubin VV, Evstigneeva ZG, Kretovich WL. 1982. High molecular weight pea leaf protein similar to the GroE protein of Escherichia coli. Biochim. Biophys. Acta 704:379-84

41. Hemmingsen SM, Woolford C, Vandervies SM, Tilly K, Dennis DT, et al. 1988. Homologous plant and bacterial proteins chaperone oligomeric protein assembly. Nature 333:330-34

42. Goloubinoff P, Gatenby AA, Lorimer GH. 1989. GroE heat-shock proteins promote assembly of foreign prokaryotic ribulose bisphosphate carboxylase oligomers in Escherichia coli. Nature 337:44-47

43. Cheng MY, Hartl FU, Horwich AL. 1990. The mitochondrial chaperonin Hsp60 is required for its own assembly. Nature 348:455-58

44. Cheng MY, Hartl FU, Martin J, Pollock RA, Kalousek F, et al. 1989. Mitochondrial heat-shock protein Hsp60 is essential for assembly of proteins imported into yeast mitochondria. Nature 337:620-25 
45. Milos P, Roy H. 1984. ATP-released large subunits participate in the assembly of RuBP carboxylase. 7. Cell Biochem. 24:153-62

46. Ostermann J, Horwich AL, Neupert W, Hartl FU. 1989. Protein folding in mitochondria requires complex formation with Hsp60 and ATP hydrolysis. Nature 341:125-30

47. Hemmingsen SM, Ellis RJ. 1986. Purification and properties of ribulosebisphosphate carboxylase large subunit binding protein. Plant Physiol. 80:269-76

48. Viitanen PV, Lubben TH, Reed J, Goloubinoff P, O'Keefe DP, Lorimer GH. 1990. Chaperoninfacilitated refolding of ribulosebisphosphate carboxylase and ATP hydrolysis by chaperonin-60 (groEL) are $\mathrm{K}^{+}$dependent. Biochemistry 29:5665-71

49. Horwich AL, Apetri AC, Fenton WA. 2009. The GroEL/GroES cis cavity as a passive anti-aggregation device. FEBS Lett. 583:2654-62

50. Priya S, Sharma SK, Sood V, Mattoo RUH, Finka A, et al. 2013. GroEL and CCT are catalytic unfoldases mediating out-of-cage polypeptide refolding without ATP. PNAS 110:7199-204

51. Clare DK, Vasishtan D, Stagg S, Quispe J, Farr GW, et al. 2012. ATP-triggered conformational changes delineate substrate-binding and -folding mechanics of the GroEL chaperonin. Cell 149:113-23

52. Goloubinoff P, Diamant S, Weiss C, Azem A. 1997. GroES binding regulates GroEL chaperonin activity under heat shock. FEBS Lett. 407:215-19

53. Ellis RJ. 1994. Molecular chaperones. Opening and closing the Anfinsen cage. Curr. Biol. 4:633-35

54. Martin J, Langer T, Boteva R, Schramel A, Horwich AL, Hartl FU. 1991. Chaperonin-mediated protein folding at the surface of groEL through a 'molten globule'-like intermediate. Nature 352:36-42

55. Buchner J, Schmidt M, Fuchs M, Jaenicke R, Rudolph R, et al. 1991. GroE facilitates refolding of citrate synthase by suppressing aggregation. Biochemistry 30:1586-91

56. Hartmann CM, Gehring H, Christen P. 1993. The mature form of imported mitochondrial proteins undergoes conformational changes upon binding to isolated mitochondria. Eur. F. Biochem. 218:905-10

57. Chaudhuri TK, Farr GW, Fenton WA, Rospert S, Horwich AL. 2001. GroEL/GroES-mediated folding of a protein too large to be encapsulated. Cell 107:235-46

58. Skowyra D, Georgopoulos C, Zylicz M. 1990. The E. coli dnaK gene product, the hsp70 homolog, can reactivate heat-inactivated RNA polymerase in an ATP hydrolysis-dependent manner. Cell 62:939-44

59. Langer T, Pfeifer G, Martin J, Baumeister W, Hartl FU. 1992. Chaperonin-mediated protein folding: GroES binds to one end of the GroEL cylinder, which accommodates the protein substrate within its central cavity. EMBO F. 11:4757-65

60. Skowyra D, Wickner S. 1993. The interplay of the GrpE heat shock protein and $\mathrm{Mg}^{2+}$ in RepA monomerization by DnaJ and DnaK. 7. Biol. Chem. 268:25296-301

61. Natalello A, Mattoo RUH, Priya S, Sharma SK, Goloubinoff P, Doglia SM. 2013. Biophysical characterization of two different stable misfolded monomeric polypeptides that are chaperone-amenable substrates. 7. Mol. Biol. 425:1158-71

62. Priya S, Sharma SK, Goloubinoff P. 2013. Molecular chaperones as enzymes that catalytically unfold misfolded polypeptides. FEBS Lett. 587:1981-87

63. Glover JR, Lindquist S. 1998. Hsp104, Hsp70, and Hsp40: a novel chaperone system that rescues previously aggregated proteins. Cell $94: 73-82$

64. Slepenkov SV, Witt SN. 2002. The unfolding story of the Escherichia coli Hsp70 DnaK: Is DnaK a holdase or an unfoldase? Mol. Microbiol. 45:1197-206

65. Mattoo RH, Goloubinoff P. 2014. Molecular chaperones are nanomachines that catalytically unfold misfolded and alternatively folded proteins. Cell. Mol. Life Sci. 71:3311-25

66. Netzer WJ, Hartl FU. 1998. Protein folding in the cytosol: chaperonin-dependent and -independent mechanisms. Trends Biochem. Sci. 23:68-73

67. Kampinga HH, Garrido C. 2012. HSPBs: small proteins with big implications in human disease. Int. $\mathcal{F}$. Biochem. Cell Biol. 44:1706-10

68. Shtilerman M, Lorimer GH, Englander SW. 1999. Chaperonin function: folding by forced unfolding. Science 284:822-25

69. Lin Z, Madan D, Rye HS. 2008. GroEL stimulates protein folding through forced unfolding. Nat. Struct. Mol. Biol. 15:303-11
(50) Reported that GroEL and CCT have intrinsic unfoldase activities but require ATP to evict oversticky intermediates.

(57) Provided experimental evidence that encapsulation is not obligate to chaperonin mechanism.

(58) Reported that Hsp70s are molecular chaperones that use ATP to convert inactive polypeptides into native proteins.

(63) Provided experimental evidence that a chaperone can disaggregate and refold stable preformed protein aggregates.

(69) Showed by Förster resonance energy transfer spectroscopy that GroEL has an intrinsic unfoldase activity. 
(70) Showed by nuclear magnetic resonance spectroscopy that GroEL has an intrinsic unfoldase activity.

(73) Reported that Hsp70 uses ATP to catalytically unfold stable misfolded proteins; their subsequent native refolding is spontaneous.

(76) Provided evidence that Hsp70 chaperones can unfold misfolded polypeptide substrates by cooperative entropic pulling.

(89) Showed that GroEL chaperonins act upon their substrate by causing local unfolding with an iterative annealing mechanism.
70. Libich DS, Tugarinov V, Clore GM. 2015. Intrinsic unfoldase/foldase activity of the chaperonin GroEL directly demonstrated using multinuclear relaxation-based NMR. PNAS 112:8817-23

71. Schroder H, Langer T, Hartl FU, Bukau B. 1993. Dnak, Dnaj and Grpe form a cellular chaperone machinery capable of repairing heat-induced protein damage. EMBO F. 12:4137-44

72. Rudiger S, Germeroth L, Schneider-Mergener J, Bukau B. 1997. Substrate specificity of the DnaK chaperone determined by screening cellulose-bound peptide libraries. EMBO F. 16:1501-7

73. Sharma SK, De Los Rios P, Christen P, Lustig A, Goloubinoff P. 2010. The kinetic parameters and energy cost of the Hsp70 chaperone as a polypeptide unfoldase. Nat. Chem. Biol. 6:914-20

74. Mattoo RUH, Sharma SK, Priya S, Finka A, Goloubinoff P. 2013. Hsp110 is a bona fide chaperone using ATP to unfold stable misfolded polypeptides and reciprocally collaborate with Hsp70 to solubilize protein aggregates. 7. Biol. Chem. 288:21399-411

75. Clerico EM, Tilitsky JM, Meng W, Gierasch LM. 2015. How Hsp70 molecular machines interact with their substrates to mediate diverse physiological functions. 7. Mol. Biol. 427:1575-88

76. De Los Rios P, Ben-Zvi A, Slutsky O, Azem A, Goloubinoff P. 2006. Hsp70 chaperones accelerate protein translocation and the unfolding of stable protein aggregates by entropic pulling. PNAS 103:6166-71

77. Vembar SS, Jonikas MC, Hendershot LM, Weissman JS, Brodsky JL. 2010. J domain co-chaperone specificity defines the role of BiP during protein translocation. F. Biol. Chem. 285:22484-94

78. Griesemer M, Young C, Robinson AS, Petzold L. 2014. BiP clustering facilitates protein folding in the endoplasmic reticulum. PLOS Comput. Biol. 10:e1003675

79. Tiwari S, Kumar V, Jayaraj GG, Maiti S, Mapa K. 2013. Unique structural modulation of a non-native substrate by cochaperone DnaJ. Biochemistry 52:1011-18

80. Kellner R, Hofmann H, Barducci A, Wunderlich B, Nettels D, Schuler B. 2014. Single-molecule spectroscopy reveals chaperone-mediated expansion of substrate protein. PNAS 111:13355-60

81. Goldstein LE, Muffat JA, Cherny RA, Moir RD, Ericsson MH, et al. 2003. Cytosolic $\beta$-amyloid deposition and supranuclear cataracts in lenses from people with Alzheimer's disease. Lancet 361:1258-65

82. Muchowski PJ, Clark JI. 1998. ATP-enhanced molecular chaperone functions of the small heat shock protein human $\alpha \mathrm{B}$ crystallin. PNAS 95:1004-9

83. Biswas A, Das KP. 2004. Role of ATP on the interaction of $\alpha$-crystallin with its substrates and its implications for the molecular chaperone function. F. Biol. Chem. 279:42648-57

84. Ghosh JG, Houck SA, Doneanu CE, Clark JI. 2006. The $\beta 4-\beta 8$ groove is an ATP-interactive site in the $\alpha$ crystallin core domain of the small heat shock protein, human $\alpha \mathrm{B}$ crystallin. 7 . Mol. Biol. 364:364-75

85. Giese KC, Vierling E. 2002. Changes in oligomerization are essential for the chaperone activity of a small heat shock protein in vivo and in vitro. 7. Biol. Chem. 277:46310-18

86. Stengel F, Baldwin AJ, Painter AJ, Jaya N, Basha E, et al. 2010. Quaternary dynamics and plasticity underlie small heat shock protein chaperone function. PNAS 107:2007-12

87. Veinger L, Diamant S, Buchner J, Goloubinoff P. 1998. The small heat-shock protein IbpB from Escherichia coli stabilizes stress-denatured proteins for subsequent refolding by a multichaperone network. 7. Biol. Chem. 273:11032-37

88. Lee GJ, Roseman AM, Saibil HR, Vierling E. 1997. A small heat shock protein stably binds heatdenatured model substrates and can maintain a substrate in a folding-competent state. EMBO 7. 16:65971

89. Todd MJ, Lorimer GH, Thirumalai D. 1996. Chaperonin-facilitated protein folding: optimization of rate and yield by an iterative annealing mechanism. PNAS 93:4030-35

90. Wiech H, Buchner J, Zimmermann R, Jakob U. 1992. Hsp90 chaperones protein folding in vitro. Nature 358:169-70

91. Rohl A, Rohrberg J, Buchner J. 2013. The chaperone Hsp90: changing partners for demanding clients. Trends Biochem. Sci. 38:253-62

92. Hartl FU, Bracher A, Hayer-Hartl M. 2011. Molecular chaperones in protein folding and proteostasis. Nature 475:324-32

93. Walerych D, Gutkowska M, Klejman MP, Wawrzynow B, Tracz Z, et al. 2010. ATP binding to Hsp90 is sufficient for effective chaperoning of p53 protein. 7. Biol. Chem. 285:32020-28 
94. Ziemienowicz A, Zylicz M, Floth C, Hubscher U. 1995. Calf thymus Hsc70 protein protects and reactivates prokaryotic and eukaryotic enzymes. F. Biol. Chem. 270:15479-84

95. Diamant S, Ben-Zvi AP, Bukau B, Goloubinoff P. 2000. Size-dependent disaggregation of stable protein aggregates by the DnaK chaperone machinery. F. Biol. Chem. 275:21107-13

96. Zolkiewski M. 1999. ClpB cooperates with DnaK, DnaJ, and GrpE in suppressing protein aggregation. A novel multi-chaperone system from Escherichia coli. 7. Biol. Chem. 274:28083-86

97. Goloubinoff P, Mogk A, Ben Zvi AP, Tomoyasu T, Bukau B. 1999. Sequential mechanism of solubilization and refolding of stable protein aggregates by a bichaperone network. PNAS 96:13732-37

98. Sauer RT, Baker TA. 2011. AAA+ proteases: ATP-fueled machines of protein destruction. Annu. Rev. Biochem. 80:587-612

99. Carroni M, Kummer E, Oguchi Y, Wendler P, Clare DK, et al. 2014. Head-to-tail interactions of the coiled-coil domains regulate $\mathrm{ClpB}$ activity and cooperation with Hsp70 in protein disaggregation. eLife $3: \mathrm{e} 02481$

100. Seyffer F, Kummer E, Oguchi Y, Winkler J, Kumar M, et al. 2012. Hsp70 proteins bind Hsp100 regulatory M domains to activate AAA plus disaggregase at aggregate surfaces. Nat. Struct. Mol. Biol. 19:1347-55

101. Aubin-Tam ME, Olivares AO, Sauer RT, Baker TA, Lang MJ. 2011. Single-molecule protein unfolding and translocation by an ATP-fueled proteolytic machine. Cell 145:257-67

102. Zolkiewski M. 2006. A camel passes through the eye of a needle: protein unfolding activity of Clp ATPases. Mol. Microbiol. 61:1094-100

103. Weibezahn J, Tessarz P, Schlieker C, Zahn R, Maglica Z, et al. 2004. Thermotolerance requires refolding of aggregated proteins by substrate translocation through the central pore of ClpB. Cell 119:653-65

104. Iosefson O, Nager AR, Baker TA, Sauer RT. 2015. Coordinated gripping of substrate by subunits of a AAA+ proteolytic machine. Nat. Chem. Biol. 11:201-6

105. Shorter J. 2011. The mammalian disaggregase machinery: Hsp110 synergizes with Hsp70 and Hsp40 to catalyze protein disaggregation and reactivation in a cell-free system. PLOS ONE 6:e26319

106. Rampelt H, Kirstein-Miles J, Nillegoda NB, Chi K, Scholz SR, et al. 2012. Metazoan Hsp70 machines use Hsp110 to power protein disaggregation. EMBO 7. 31:4221-35

107. Schuermann JP, Jiang J, Cuellar J, Llorca O, Wang L, et al. 2008. Structure of the Hsp110:Hsc70 nucleotide exchange machine. Mol. Cell 31:232-43

108. Torrente MP, Shorter J. 2014. The metazoan protein disaggregase and amyloid depolymerase system: Hsp110, Hsp70, Hsp40, and small heat shock proteins. Prion 7:457-63

109. Ebrahimi-Fakhari D, Wahlster L, McLean PJ. 2012. Protein degradation pathways in Parkinson's disease: curse or blessing. Acta Neuropathol. 124:153-72

110. Zempel H, Mandelkow E. 2014. Lost after translation: missorting of Tau protein and consequences for Alzheimer disease. Trends Neurosci. 37:721-32

111. Farr GW, Fenton WA, Chaudhuri TK, Clare DK, Saibil HR, Horwich AL. 2003. Folding with and without encapsulation by cis- and trans-only GroEL-GroES complexes. EMBO 7. 22:3220-30

112. Weiss C, Goloubinoff P. 1995. A mutant at position 87 of the GroEL chaperonin is affected in protein binding and ATP hydrolysis. F. Biol. Chem. 270:13956-60

113. Azia A, Unger R, Horovitz A. 2012. What distinguishes GroEL substrates from other Escherichia coli proteins? FEBS 7. 279:543-50

114. Rudiger S, Schneider-Mergener J, Bukau B. 2001. Its substrate specificity characterizes the DnaJ cochaperone as a scanning factor for the DnaK chaperone. EMBO 7. 20:1042-50

115. Stan G, Thirumalai D, Lorimer GH, Brooks BR. 2003. Annealing function of GroEL: structural and bioinformatic analysis. Biophys. Chem. 100:453-67

116. Tokuriki N, Tawfik DS. 2009. Chaperonin overexpression promotes genetic variation and enzyme evolution. Nature 459:668-73

117. Bandyopadhyay A, Saxena K, Kasturia N, Dalal V, Bhatt N, et al. 2012. Chemical chaperones assist intracellular folding to buffer mutational variations. Nat. Chem. Biol. 8:238-45

118. Jarosz DF, Lindquist S. 2010. Hsp90 and environmental stress transform the adaptive value of natural genetic variation. Science 330:1820-24 
119. de Los Rios P, Goloubinoff P. 2012. Protein folding chaperoning protein evolution. Nat. Chem. Biol. 8:226-28

120. Finka A, Mattoo RUH, Goloubinoff P. 2011. Meta-analysis of heat- and chemically upregulated chaperone genes in plant and human cells. Cell Stress Chaperones 16:15-31

121. Wolff S, Weissman JS, Dillin A. 2014. Differential scales of protein quality control. Cell 157:52-64

122. Hinault MP, Goloubinoff P. 2007. Molecular crime and cellular punishment: active detoxification of misfolded and aggregated proteins in the cell by the chaperone and protease networks. Adv. Exp. Med. Biol. 594:47-54

123. Brehme M, Voisine C, Rolland T, Wachi S, Soper JH, et al. 2014. A chaperome subnetwork safeguards proteostasis in aging and neurodegenerative disease. Cell Rep. 9:1135-50

124. Labbadia J, Morimoto RI. 2015. The biology of proteostasis in aging and disease. Annu. Rev. Biochem. $84: 435-64$

125. Johnson KA. 2010. The kinetic and chemical mechanism of high-fidelity DNA polymerases. Biochim. Biophys. Acta 1804:1041-48

126. Fasken MB, Corbett AH. 2005. Process or perish: quality control in mRNA biogenesis. Nat. Struct. Mol. Biol. 12:482-88

127. Yadavalli SS, Ibba M. 2012. Quality control in aminoacyl-tRNA synthesis: its role in translational fidelity. Adv. Protein Chem. Struct. Biol. 86:1-43

128. Hinault MP, Cuendet AFH, Mattoo RUH, Mensi M, Dietler G, et al. 2010. Stable $\alpha$-synuclein oligomers strongly inhibit chaperone activity of the Hsp70 system by weak interactions with J-domain co-chaperones. F. Biol. Chem. 285:38173-82

129. Doyle SM, Genest O, Wickner S. 2013. Protein rescue from aggregates by powerful molecular chaperone machines. Nat. Rev. Mol. Cell. Biol. 14:617-29

130. Bromberg Z, Goloubinoff P, Saidi Y, Weiss YG. 2013. The membrane-associated transient receptor potential vanilloid channel is the central heat shock receptor controlling the cellular heat shock response in epithelial cells. PLOS ONE 8:e57149

131. Finka A, Cuendet AFH, Maathuis FJM, Saidi Y, GoloubinoffP. 2012. Plasma membrane cyclic nucleotide gated calcium channels control land plant thermal sensing and acquired thermotolerance. Plant Cell 24:3333-48

132. Whitesell L, Santagata S, Lin NU. 2012. Inhibiting Hsp90 to treat cancer: a strategy in evolution. Curr. Mol. Med. 12:1108-24

133. McConnell JR, McAlpine SR. 2013. Heat shock proteins 27, 40, and 70 as combinational and dual therapeutic cancer targets. Bioorg. Med. Chem. Lett. 23:1923-28

134. Saidi Y, Finka A, Muriset M, Bromberg Z, Weiss YG, et al. 2009. The heat shock response in moss plants is regulated by specific calcium-permeable channels in the plasma membrane. Plant Cell 21:2829-43

135. Mittler R, Finka A, Goloubinoff P. 2012. How do plants feel the heat? Trends Biochem. Sci. 37:118-25

136. Saidi Y, Finka A, Goloubinoff P. 2011. Heat perception and signalling in plants: a tortuous path to thermotolerance. New Phytol. 190:556-65

137. Kampinga HH, Hageman J, Vos MJ, Kubota H, Tanguay RM, et al. 2009. Guidelines for the nomenclature of the human heat shock proteins. Cell Stress Chaperones 14:105-11

138. Karagoz GE, Duarte AMS, Akoury E, Ippel H, Biernat J, et al. 2014. Hsp90-Tau complex reveals molecular basis for specificity in chaperone action. Cell 156:963-74 
$\mathrm{A}$
$\mathrm{R}$

\section{Contents}

Annual Review of Biochemistry

Volume 85, 2016

Cellular Homeostasis and Aging

F. Ulrich Hartl

Dietary Protein, Metabolism, and Aging

George A. Soultoukis and Linda Partridge 5

Signaling Networks Determining Life Span

Celine E. Riera, Carsten Merkwirth, C. Daniel De Magalhaes Filho, and Andrew Dillin

Mitochondrial Gene Expression: A Playground of Evolutionary

Tinkering

Walter Neupert

Organization and Regulation of Mitochondrial Protein Synthesis

Martin Ott, Alexey Amunts, and Alan Brown

Structure and Function of the Mitochondrial Ribosome Basil 7. Greber and Nenad Ban

Maintenance and Expression of Mammalian Mitochondrial DNA

Claes M. Gustafsson, Maria Falkenberg, and Nils-Göran Larsson

Enjoy the Trip: Calcium in Mitochondria Back and Forth

Diego De Stefani, Rosario Rizzuto, and Tullio Pozzan

Mechanics and Single-Molecule Interrogation of DNA Recombination

Fason C. Bell and Stephen C. Kowalczykowski

CRISPR/Cas9 in Genome Editing and Beyond

Haifeng Wang, Marie La Russa, and Lei S. Qi

Nucleotide Excision Repair and Transcriptional Regulation: TFIIH

and Beyond

Emmanuel Compe and fean-Marc Egly

Transcription as a Threat to Genome Integrity

Hélène Gaillard and Andrés Aguilera .....

Mechanisms of Bacterial Transcription Termination: All Good Things

Must End

Ananya Ray-Soni, Michael 7. Bellecourt, and Robert Landick.... 
Nucleic Acid-Based Nanodevices in Biological Imaging

Kasturi Chakraborty, Aneesh T. Veetil, Samie R. Jaffrey, and Yamuna Krishnan ..... 349

The p53 Pathway: Origins, Inactivation in Cancer, and Emerging

Therapeutic Approaches

Andreas C. Foerger and Alan R. Fersht

The Substrate Specificity of Sirtuins

Poonam Bheda, Hui Fing, Cynthia Wolberger, and Hening Lin

Macrodomains: Structure, Function, Evolution, and Catalytic Activities

Jobannes Gregor Matthias Rack, Dragutin Perina, and Ivan Abel

Biosynthesis of the Metalloclusters of Nitrogenases

Yilin Hu and Markus W. Ribbe

Radical S-Adenosylmethionine Enzymes in Human Health and Disease

Bradley 7. Landgraf, Erin L. McCarthy, and Squire 7. Booker ....

Ice-Binding Proteins and Their Function

Maya Bar Dolev, Ido Braslavsky, and Peter L. Davies.

Shared Molecular Mechanisms of Membrane Transporters

David Drew and Olga Boudker

Spatial and Temporal Regulation of Receptor Tyrosine Kinase

Activation and Intracellular Signal Transduction

fobn 7.M. Bergeron, Gianni M. Di Guglielmo, Sophie Dahan,

Michel Dominguez, and Barry I. Posner

Understanding the Chemistry and Biology of Glycosylation with

Glycan Synthesis

Larissa Krasnova and Chi-Huey Wong

The Biochemistry of $O$-GlcNAc Transferase: Which Functions Make

It Essential in Mammalian Cells?

Zebulon G. Levine and Suzanne Walker

Mechanisms of Mitotic Spindle Assembly

Sabine Petry

Mammalian Autophagy: How Does It Work?

Carla F. Bento, Maurizio Renna, Gbita Ghislat, Claudia Puri, Avrabam Ashkenazi,

Mariella Vicinanza, Fiona M. Menzies, and David C. Rubinsztein

Experimental Milestones in the Discovery of Molecular Chaperones as Polypeptide Unfolding Enzymes Andrija Finka, Rayees U.H. Mattoo, and Pierre Goloubinoff

Necroptosis and Inflammation

Kim Newton and Gerard Manning 
Reactive Oxygen Species and Neutrophil Function

Christine C. Winterbourn, Anthony 7. Kettle, and Mark B. Hampton

\section{Indexes}

Cumulative Index of Contributing Authors, Volumes 81-85 _.................... 793

Cumulative Index of Article Titles, Volumes 81-85 _............................ 797

\section{Errata}

An online log of corrections to Annual Review of Biochemistry articles may be found at http://www.annualreviews.org/errata/biochem 